\title{
AGN wind scaling relations and the co-evolution of black holes and galaxies
}

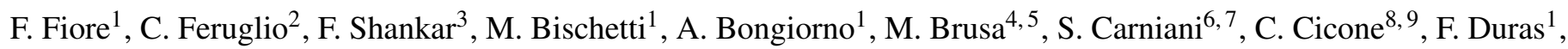
A. Lamastra ${ }^{1}$, V. Mainieri ${ }^{10}$, A. Marconi ${ }^{6,11}$, N. Menci ${ }^{1}$, R. Maiolino ${ }^{7}$, E. Piconcelli ${ }^{1}$, G. Vietri ${ }^{1}$, and L. Zappacosta ${ }^{1}$

1 INAF-Osservatorio Astronomico di Roma, via Frascati 33, 00078 Monteporzio Catone, Italy

e-mail: fabrizio.fiore@oa-roma.inaf.it

2 INAF-Osservatorio Astronomico di Trieste, via G. Tiepolo 11, 34124 Trieste, Italy

3 Department of Physics and Astronomy, University of Southampton, Highfield, SO17 1BJ, UK

${ }^{4}$ Dipartimento di Fisica e Astronomia, Alma Mater Studiorum - Universitá di Bologna, viale Berti Pichat 6/2, 40127 Bologna, Italy

5 INAF-Osservatorio Astronomico di Bologna, via Ranzani 1, 40127 Bologna, Italy

6 Dipartimento di Fisica e Astronomia, Universitá di Firenze, via G. Sansone 1, 50019 Sesto F.no, Firenze, Italy

7 Cavendish Laboratory, University of Cambridge, 19 J. J. Thomson Ave., Cambridge CB3 0HE, UK

8 INAF-Osservatorio Astronomico di Brera, via Brera 28, 20121 Milan, Italy

9 ETH, Institute for Astronomy, Department of Physics, Wolfgang-Pauli-Strasse 278093 Zurich, Switzerland

${ }_{10}$ European Southern Observatory, Karl-Schwarzschild-str. 2, 85748 Garching bei München, Germany

11 INAF-Osservatorio Astrofisico di Arcetri, Largo E. Fermi 5, 50125 Firenze, Italy

Received 4 August 2016 / Accepted 13 February 2017

\begin{abstract}
Context. Feedback from accreting supermassive black holes (SMBHs) is often identified as the main mechanism responsible for regulating star formation in active galactic nucleus (AGN) host galaxies. However, the relationships between AGN activity, radiation, winds, and star formation are complex and still far from being understood.

Aims. We study scaling relations between AGN properties, host galaxy properties, and AGN winds. We then evaluate the wind mean impact on the global star formation history, taking into account the short AGN duty cycle with respect to that of star formation.

Methods. We first collect AGN wind observations for 94 AGN with detected massive winds at sub-pc to kpc spatial scales. We then fold AGN wind scaling relations with AGN luminosity functions, to evaluate the average AGN wind mass-loading factor as a function of cosmic time.

Results. We find strong correlations between the AGN molecular and ionised wind mass outflow rates and the AGN bolometric luminosity. The power law scaling is steeper for ionised winds (slope $1.29 \pm 0.38$ ) than for molecular winds $(0.76 \pm 0.06)$, meaning that the two rates converge at high bolometric luminosities. The molecular gas depletion timescale and the molecular gas fraction of galaxies hosting powerful AGN driven winds are 3-10 times shorter and smaller than those of main sequence galaxies with similar star formation rate (SFR), stellar mass, and redshift. These findings suggest that, at high AGN bolometric luminosity, the reduced molecular gas fraction may be due to the destruction of molecules by the wind, leading to a larger fraction of gas in the atomic ionised phase. The AGN wind mass-loading factor $\eta=\dot{M}_{\mathrm{OF}} / \mathrm{SFR}$ is systematically higher than that of starburst driven winds.

Conclusions. Our analysis shows that AGN winds are, on average, powerful enough to clean galaxies from their molecular gas only in massive systems at $z \lesssim 2$, i.e. a strong form of co-evolution between SMBHs and galaxies appears to break down for the least massive galaxies.
\end{abstract}

Key words. galaxies: active - galaxies: evolution - quasars: general

\section{Introduction}

The past decades have seen a hot debate on whether, and how, the evolution of galaxies and of the supermassive black holes (SMBHs) hosted in their nuclei is correlated.

The debate started with the HST discovery of SMBHs in most local bulges (Richstone et al. 1998). SMBH mass and host bulge properties - such as velocity dispersion, luminosity, and mass - were found to closely correlate with each other (Gebhardt et al. 2000; Ferrarese \& Ford 2005; Kormendy \& Ho 2013, and references therein, but see also Shankar et al. 2016, 2017). Furthermore, the comparison of the SMBH mass function derived from the active galactic nucleus (AGN) luminosity function and from the local bulge luminosity function suggests that SMBH growth is mostly due to accretion of matter during their active phases, and therefore that most bulge galaxies passed a phase of strong nuclear activity (Soltan 1982; Marconi et al. 2004; Shankar et al. 2004; Merloni \& Heinz 2008). Both findings seemed to imply links between SMBH accretion and bulge formation, i.e. a strong form of AGN/galaxy co-evolution. Indeed, soon after the discovery of the SMBH-bulge relationships, several authors (Silk \& Rees 1998; Fabian 1999; King 2003; Granato et al. 2004) suggested that they can be naturally explained if AGN winds efficiently interact with the galaxy ISM. When the black hole reaches a critical mass it may be powerful enough to heat up and eject the gas from the galaxy, terminating the growth of both SMBH and galaxy, and giving rise to the observed scaling between SMBH mass and bulge velocity dispersion. AGN feedback not only modify AGN host galaxies it can also affect the intra-cluster matter (ICM) in groups and 
clusters of galaxies. Two modes for AGN feedback have been indeed postulated, the so-called radio-mode in the central cluster galaxies and the quasar-mode, characterised by slower winds of both ionised, neutral atomic, and molecular matter.

Radio-mode feedback is evident in cool core clusters and groups, where the ICM is heated up by AGN jet-driven radio bubbles. The power to excavate cavities in the ICM is proportional to the X-ray luminosity, and the power in cavities is proportional to the AGN radio luminosity (see McNamara \& Nulsen 2007; Cattaneo et al. 2009; Fabian 2012, for reviews). Interestingly, only the brightest central galaxies (BCGs) in clusters/groups with low inner entropy (short cooling time) have an active nucleus, and are actively forming stars (Cavagnolo et al. 2008, 2009). The situation is best described by Voit \& Donahue (2015): "a delicate feedback mechanism where AGN input energy regulates the gas entropy and in turn further gas accretion and star formation (stars can form from low entropy, cold and dense gas only)". Thus, a multiphase gas structure naturally develops in cluster cores and within the BCGs leading to AGN feedback triggered by cold accretion (Gaspari et al. 2012, 2013, 2014, 2017).

Similar autoregulation may occur in galaxies other than BCGs, where feedback might be due to more common AGN winds. Indeed, several direct observation of ISM modifications by AGN winds have been collected so far. Cano-Diaz et al. (2012), Cresci et al. (2015), and Carniani et al. (2016) have found that AGN winds and actively star-forming regions are spatially anti-correlated. Similarly, Davies et al. (2007) and Lipari et al. (2009) found little evidence for young (Myr) stellar populations in the $\lesssim 1 \mathrm{kpc}$ region of Markarian 231 where a powerful molecular outflow is observed (Feruglio et al. 2010, 2015). However, although promising, these quasar-mode feedback observations are still too sparse to derive strong conclusions. The correlation between SMBHs and bulge properties do not necessarily require feedback, and can be also explained if SMBHs and bulges formed simultaneously, during episodes when a fixed fraction of gas accretes toward the central black hole while the rest forms the spheroid stars. Menci et al. (2003) reproduced the $\mathrm{BH}$ mass $-\sigma_{\text {bulge }}$ correlation as the combination of three factors: a) the merging histories of the galactic dark matter clumps, implying that the mass of the available cold gas scales as $\sigma^{2.5}$; b) the destabilisation of cold gas by galaxy interactions, which steepens the correlation by another factor $\sigma$; and c) $\mathrm{SNe}$ feedback, which depletes the residual gas content of shallow potential wells, further steepening the correlation. Later, Peng (2007) showed that galaxy mergers are efficient in averaging out extreme values of $M_{\mathrm{BH}} / M_{*}$, converging toward a narrow correlation between these quantities, close to the observed one, even starting from arbitrary distributions. Jahnke \& Macció (2011) showed that the number of mergers needed to this purpose is consistent with that of standard merger tree models of hierarchical galaxy (and SMBH) formation. In this scenario the SMBHs and bulges do not necessarily know about each other. No causal connection exists between these systems, and their properties are connected just by natural scaling relations. We can call this as a weak form of AGN/galaxy co-evolution. More recently, the analysis of Shankar et al. (2016) supports a strong dependence between SMBH mass and bulge velocity dispersion, while the dependence with the bulge mass is weaker, disfavouring this scenario, and suggesting to investigate AGN/galaxy co-evolution independently from the SMBH mass - bulge mass scaling relations.

Comparing model predictions to the observed SMBH mass bulge properties hardly allows one to discriminate between weak and strong forms of AGN/galaxy co-evolution. This is probably due to the fact that SMBH mass and bulge properties are quantities integrated along cosmic time, with SMBHs and bulges assembled during the Hubble time, as a consequence of several merging and accretion events. A different route attempted to distinguish between weak and strong forms of co-evolution, is to study derivative quantities, such as the SMBH accretion rate and the star formation rate (SFR), or, the cosmological evolution of the AGN and galaxy luminosity densities. Franceschini et al. (1999) were among the first to realise that the luminosity dependent evolution of AGN, with lower luminosity AGN peaking at a redshift lower than luminous QSOs (Ueda et al. 2003, 2014; Fiore et al. 2003; La Franca et al. 2005; Brandt \& Hasinger 2005; Bongiorno et al. 2007; Aird et al. 2015; Brandt $\&$ Alexander 2015), mirrors that of star-forming galaxies and of massive spheroids. These trends, dubbed "downsizing" by Cowie et al. (1996), and in general the relationship between the evolution of AGN and galaxy growth, may arise from feedback mechanisms linking nuclear and galactic processes.

Indirect evidence for AGN feedback come from the statistical properties of AGN host galaxies with respect to the inactive population. It is well known since the pioneering HST studies of Bahcall et al. (1997) that luminous QSOs reside preferentially in massive, spheroid-dominated host galaxies, whereas lower luminosity QSOs are found in both spheroidal and disky galaxies (Dunlop et al. 2003; Jahnke et al. 2004, and references therein). The distribution of AGN host galaxy colours, morphologies, SFR, specific SFR are wider than that of star-forming galaxies of similar masses, and skewed toward redder/more inactive galaxies (e.g. Alexander et al. 2002; Mignoli et al. 2004; Brusa et al. 2005, 2009, 2010; Nandra et al. 2007; Mainieri et al. 2011; Bongiorno et al. 2012; Georgakakis et al. 2014). Many AGN are hosted in red-and-dead galaxies, or lie in the so called green valley. Recent ALMA observations of X-ray selected AGN in the GOODS field (Mullaney et al. 2015) confirmed these earlier results, showing that the bulk of the AGN population lie below the galaxy main sequence (see Daddi et al. 2007; Rodighiero et al. 2011, and refs. therein). Because the stellar mass function of star-forming galaxies is exponentially cut-offed above a quenching mass $M_{*} \sim 10^{11} M_{\odot}$ (Peng et al. 2010), the galaxy main sequence flattens above the same mass, whereas the star formation efficiency and the gas-to-star mass fraction decrease (Genzel et al. 2010, and references therein). AGN feedback may well be one of the drivers of these transformations, as well as the main driver for the quenching of star formation in massive galaxies (Bongiorno et al. 2016), pointing toward a strong form of AGN/galaxy co-evolution. We explore this possibility in this paper.

This paper is organised as follows. In Sect. 2 we review AGN massive wind observations, and study the scaling relationships between wind mass outflow rate, velocity, kinetic power, momentum load, AGN bolometric luminosity and host galaxy SFR. We then plug AGN wind studies in the broader scenario of starforming galaxies scaling relations (Genzel et al. 2015, and references therein), to understand whether AGN hosting strong winds are outliers in these relationships. We study the relationships between the depletion timescale (the ratio between molecular gas mass and SFR), and gas fraction (the ratio between molecular gas mass and galaxy stellar mass), with the offset from the galaxy main sequence, redshift and host galaxy stellar mass, for a sample of sources with interferometric molecular measurements. In Sect. 3 we evaluate the wind statistical relevance on the global star formation history, by folding the AGN wind scaling relations with the AGN luminosity functions. This accounts for the fact that AGN shine in a relatively small fraction of galaxies, i.e. 
the AGN timescales are usually shorter than the star formation timescales. We compare the cosmic, average AGN outflow rate, computed by using the AGN wind scaling relations, to the galaxy cosmic star formation rate, to study the regimes (galaxy masses, cosmic epoch) where AGN winds are statistically strong enough to affect star formation in the global galaxy population. Section 4 presents our conclusions. A $\mathrm{H}_{0}=70 \mathrm{~km} \mathrm{~s}^{-1} \mathrm{Mpc}^{-1}, \Omega_{\mathrm{M}}=0.3$, $\Omega_{\Lambda}=0.7$ cosmology is adopted throughout.

\section{AGN wind scaling relations}

Although wind observations are very common in AGN (see Elvis 2000; Veilleux et al. 2005; and Fabian 2012, for reviews), most studies concern ionised gas and uncertain spatial scales. In the past few years the situation changed drastically. Several fast $\left(v_{\mathrm{OF}}\right.$ of the order of $1000 \mathrm{~km} \mathrm{~s}^{-1}$ ), massive outflows of ionised, neutral and molecular gas, extended on kpc scales, have been discovered thanks to three techniques: 1) deep optical/NIR spectroscopy, mainly from integral field observations (IFU, e.g. Nesvadba et al. 2006, 2008; Alexander et al. 2010; Rupke \& Veilleux 2011; Riffel \& Storchi-Bergmann 2011; Cano-Diaz et al. 2012; Greene et al. 2012; Harrison et al. 2012, 2014; Liu et al. 2013a,b; Cimatti et al. 2013; Tadhunter et al. 2014; Genzel et al. 2014; Brusa et al. 2015a; Cresci et al. 2015; Carniani et al. 2015; Perna et al. 2015a,b; Zakamska et al. 2016); 2) interferometric observations in the (sub)millmetre domain (e.g. Feruglio et al. 2010, 2013a,b, 2015; Alatalo et al. 2011; Aalto et al. 2012; Cicone et al. 2012, 2014, 2015; Maiolino et al. 2012, Krips et al. 2011; Morganti et al. 2013a,b; Combes et al. 2013; Garcia-Burillo et al. 2014); and 3) far-infrared spectroscopy from Herschel (e.g. Fischer et al. 2010; Sturm et al. 2011; Veilleux et al. 2013; Spoon et al. 2013; Stone et al. 2016; Gonzalez-Alfonso et al. 2017). In addition, AGN-driven winds from the accretion disk scale up to the dusty torus are now detected routinely both in the local and in the distant Universe, as blue-shifted absorption lines in the X-ray spectra of a substantial fraction of AGN (e.g. Piconcelli et al. 2005; Kaastra et al. 2014). The most powerful of these winds, observed in $20-40 \%$ of local AGN (e.g. Tombesi et al. 2010) and in a handful of higher redshift objects (e.g. Chartas et al. 2009; Lanzuisi et al. 2012), have extreme velocities (ultrafast outflows, UFOs, v 0.1-0.3c) and are made by highly ionised gas which can be detected only at X-ray energies.

We collected from the literature observations of AGN with reliable massive outflow detections, for which there is an estimate (or a robust limit) on the physical size of the high velocity gas involved in the wind. The sample includes molecular winds, ionised winds (from [OIII], $\mathrm{H} \alpha$ and $\mathrm{H} \beta$ lines), broad absorption line (BAL) winds and X-ray absorbers (both UFOs and the slower "warm absorbers"). We give in Appendix A a short description of the source samples used in the following analysis.

We have recomputed the wind physical properties (mass outflow rate, kinetic energy rate) using the same assumptions for all sources of each sample (as detailed in Appendix B). While wind geometry, wind gas density, temperature, metallicity etc. may well differ from source to source, applying a uniform analysis strategy minimizes systematic differences from sample to sample. In fact, self-consistent information of the gas physical and chemical properties is not available for the majority of the sources with detected winds, and thus assumptions on these properties must be done in any case. For ionised wind parameters, the chain of assumptions needed to convert observed quantities into physical quantities is particularly long (see Appendix B), and therefore the largest uncertainties concern these winds (about one order of magnitude or even more, see Harrison et al. 2014). We also collected from the literature AGN and galaxy properties, such as luminosities, SFRs, stellar masses, molecular gas masses. We note that these quantities are calculated by different authors, using non-homogeneous recipes. In particular, bolometric luminosity are calculated either from fitting optical-UV spectral energy distributions (SEDs) with AGN templates and from X-ray or infrared luminosities by applying a bolometric correction. Most SFRs are calculated from far infrared luminosities and therefore are not instantaneous SFRs. Stellar masses are calculated from modelling optical-nearinfrared galaxy SEDs with galaxy templates or by converting near infrared luminosities from IFU observations of nearby AGN host galaxies into stellar masses. Molecular gas masses are calculated converting $\mathrm{CO}$ luminosities into $\mathrm{H}_{2}$ gas masses, by assuming a standard conversion factor (see Appendix for details). This unavoidably introduces some scatter in the correlations discussed in the following sections.

Altogether, we have assembled a sample of 109 wind measurements of 94 AGN with detected massive winds at different scales (sub-pc to $\mathrm{kpc}$ ) and ionisation states, that we use to constrain the relationships between wind parameters, AGN parameters and host galaxy parameters. This sample is definitely not complete and suffers from strong selection biases; above all, we note that most molecular winds and UFOs are found in local ULIRGs and Seyfert galaxies. Ionised winds are found in both low-redshift AGN and $z=2-3$ luminous/hyper-luminous QSOs. BALs are from $z=2-3$ QSOs.

\subsection{Wind parameters vs. AGN parameters}

Figure 1 shows the wind mass outflow rate (left panel) and kinetic power (right panel) as a function of the AGN bolometric luminosity. The mass outflow rate and kinetic power of molecular winds (blue symbols) are correlated rather well with the AGN bolometric luminosity (see Table 1, which gives for each correlation the Spearman rank, SR, correlation coefficient, the probability of the correlation and the best fit slope, obtained from a least square fit between the two variables). The log linear slope is $0.76 \pm 0.06$ for the mass outflow rate and $1.27 \pm 0.04$ for the kinetic power. The average ratio $\dot{E}_{\text {kin }} / L_{\text {bol }}$ in the molecular winds sample is $2.5 \%$.

Ionised winds (green symbols), BAL winds (black symbols), and X-ray absorbers (red symbols), lie below the correlation found for molecular winds. Most ionised winds have $\dot{M}_{\mathrm{OF}} 10$ 100 times smaller than molecular winds at $L_{\mathrm{bol}} \lesssim 10^{46} \mathrm{erg} / \mathrm{s}$. Above this luminosity, ionised winds have $\dot{M}_{\mathrm{OF}}$ similar or a few times lower than molecular winds. There is a good correlation between $\dot{M}_{\mathrm{OF}}, \dot{E}_{\text {kin }}$, and the bolometric luminosity for ionised winds (see Table 1) with log linear slopes $1.29 \pm 0.38$ and $1.50 \pm 0.34$ respectively. The average ratio $\dot{E}_{\text {kin }} / L_{\text {bol }}$ for the ionised winds sample is $0.16 \%$ at $\log L_{\text {bol }}=45$ and $0.30 \%$ at $\log L_{\text {bol }}=47$.

X-ray absorbers and BAL winds have respectively $\dot{M}_{\mathrm{OF}} \sim$ 500,30 times lower than what expected from the best fit linear correlation for molecular winds, again showing a trend for higher differences with respect to molecular winds at lower bolometric luminosities. About half X-ray absorbers and BAL winds have $\dot{E}_{\text {kin }} / L_{\text {bol }}$ in the range $1-10 \%$ with another half having $\dot{E}_{\text {kin }} / L_{\text {bol }}<1 \%$.

The left panel of Fig. 2 show the AGN bolometric luminosity as a function of the maximum wind velocity, $v_{\max }$, defined following Rupke \& Veilleux (2013) as the shift between the velocity peak of broad emission lines and the systemic velocity plus 2 times the $\sigma$ of the broad Gaussian component, see 

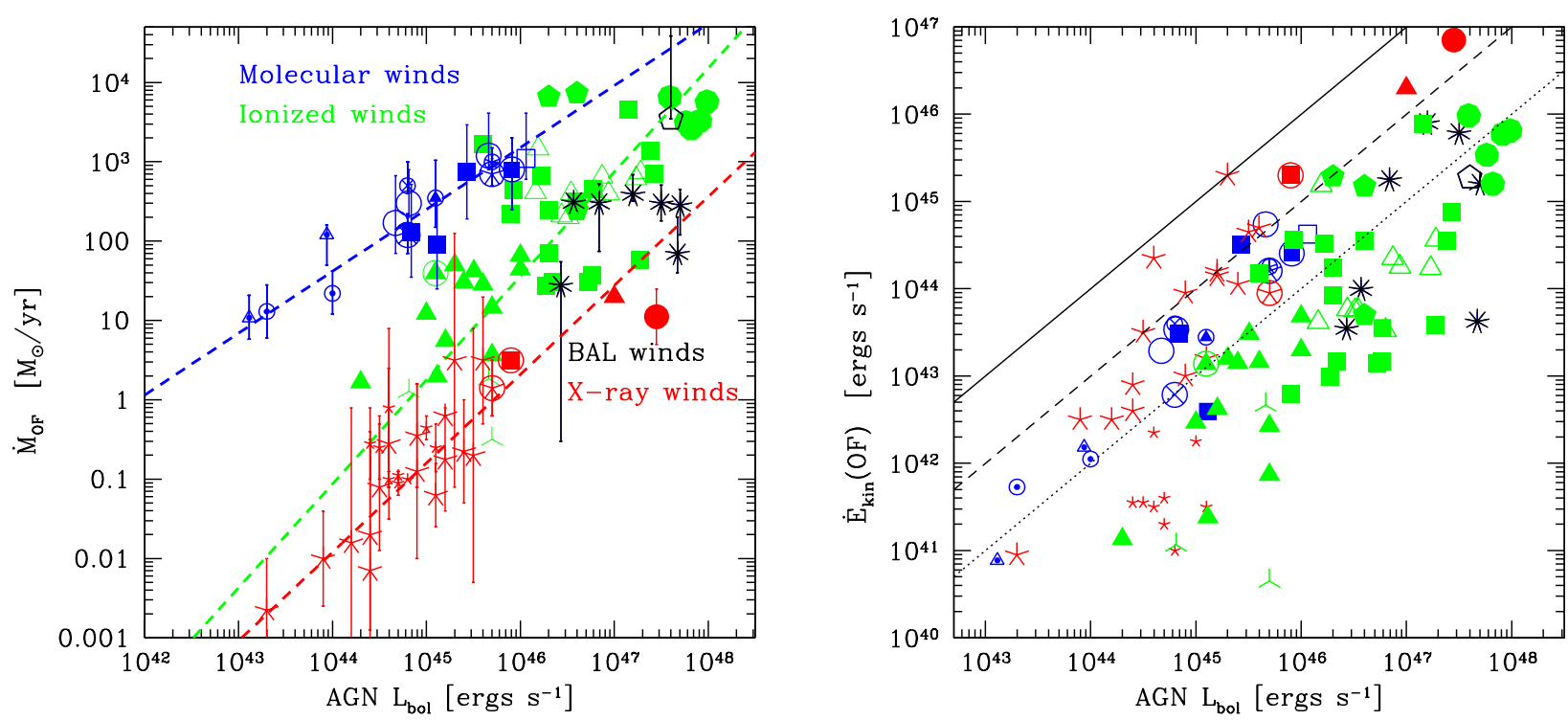

Fig. 1. Left panel: the wind mass outflow rate as a function of the AGN bolometric luminosity. AGN for which molecular winds have been reported in the literature (mostly local ULIRGs and Seyfert galaxies) are shown with blue symbols. In particular: open circles are CO outflows; the open square is the measurement for IRAS 23060; filled squares are OH outflows; the starred open circles are for Markarian 231 (large symbol for the outflow measured within $R_{\mathrm{OF}}=1 \mathrm{kpc}$ and small symbol for the outflow at $R_{\mathrm{OF}}=0.3 \mathrm{kpc}$ ); the crossed open circles are the measurements for NGC 6240 (large symbol for $R_{\mathrm{OF}}=3.5 \mathrm{kpc}$ and small symbol for $R_{\mathrm{OF}}=0.6 \mathrm{kpc}$ ); the small dotted open triangle marks the measurement in the circum nuclear disk of NGC $1068\left(R_{\mathrm{OF}}=0.1 \mathrm{kpc}\right)$ and NGC $1433\left(R_{\mathrm{OF}}=0.06 \mathrm{kpc}\right)$; the small dotted open circles represent the measurements for NGC 1266, IC 5066 at $R_{\mathrm{OF}}=0.5 \mathrm{kpc}$; the squared open circle marks IRAS F11119+13257 measurement at $R_{\mathrm{OF}}=0.3 \mathrm{kpc}$. Green symbols mark ionised outflows measurements. In details: filled squares mark $z>1 \mathrm{AGN}$; filled triangles mark $z=0.1-0.2$ AGN; open triangles mark $z=0.4-0.6$ type 2 AGN; pentagons mark $z=2-3$ radiogalaxies; filled circles mark hyper-luminous $z=2-3$ QSOs. BAL winds are shown with black stars. The black open pentagon highlights the [CII] wind in J1148+5251 at $z=6.4$. Finally, red symbols mark X-ray outflows. In details: large five pointed stars are local UFOs; the starred open circle, the filled triangle and the circled square are the measurement for Markarian 231, PDS456 and IRAS F11119+13257, respectively. Small five point stars are slower warm absorbers. The dashed blue, green and red lines are the best fit correlations of the molecular, ionised, and X-ray absorber samples, respectively. Right panel: wind kinetic power as a function of the AGN bolometric luminosity. Solid, dashed and dotted line represent the correlations $\dot{E}_{\mathrm{kin}}=1,0.1,0.01 L_{\mathrm{bol}}$.
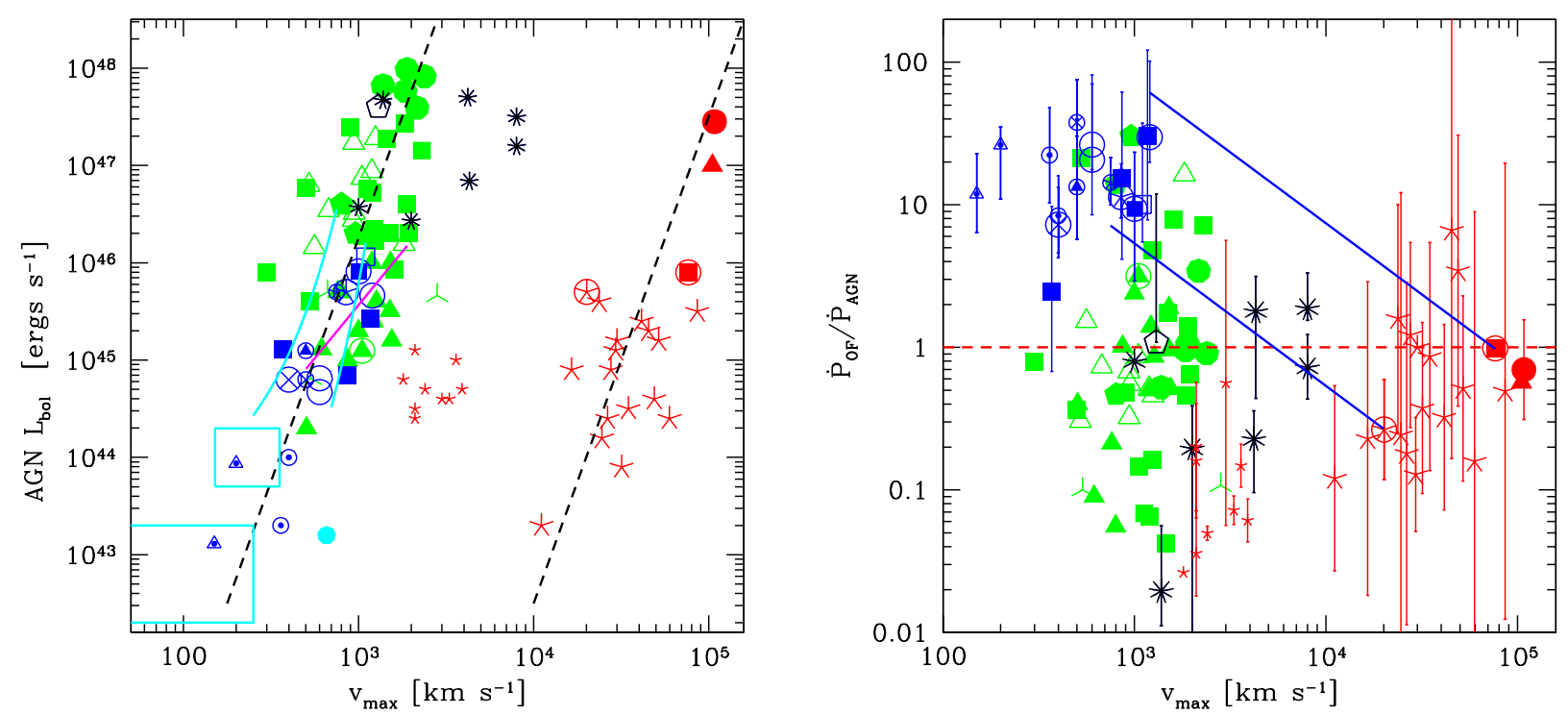

Fig. 2. Left panel: AGN bolometric luminosity as a function of the maximum wind velocity, $v_{\max }$. The black dashed lines mark a $v_{\max }^{5} \mathrm{scaling}$. The magenta solid line is the best fit correlation found by Spoon et al. (2013) for OH outflows. The two cyan solid lines are the best fit scaling found by Veilleux et al. (2013) for OH outflows, using $v_{\max }$ and $v_{80}$. The cyan boxes and filled dot are the loci covered by two groups of Swift BAT AGN with $42.3<L_{\mathrm{bol}}<43.3$ and $43.7<L_{\mathrm{bol}}<44.3$ and by the outlier NGC 7479, from Stone et al. (2016). Right panel: wind momentum load (outflow momentum rate divided by the AGN radiation momentum rate $L / c$ ) as a function of $v_{\max }$. The red dashed line mark the expectations for a momentum conserving outflow. The two blue solid lines mark the expectations for pure energy conserving outflows for Markarian 231 (starred circle) and IRAS F11119+13257 (squared circle). Symbols as in Fig. 1. 

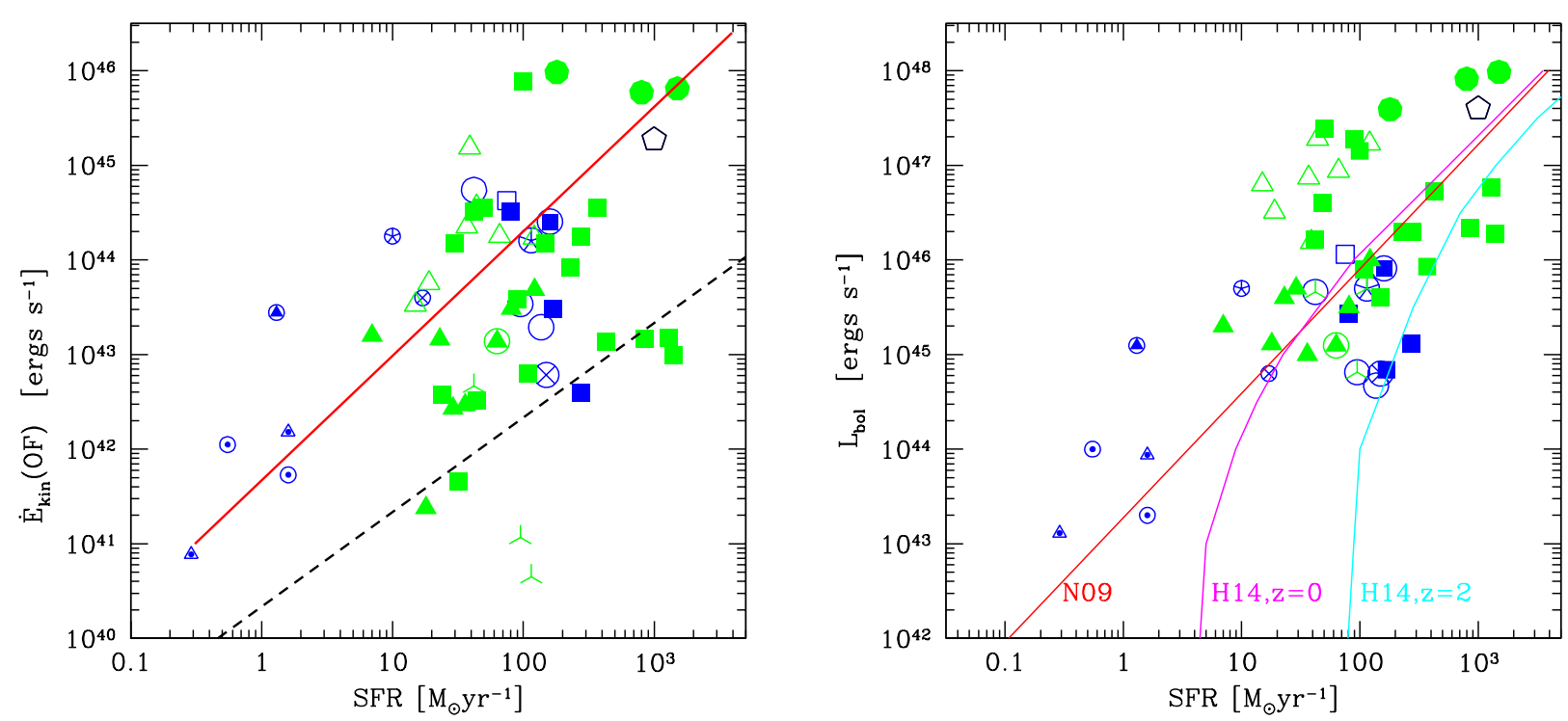

Fig. 3. Left panel: outflow kinetic power as a function of the star formation rate in the host galaxy (computed, when possible, in a region similar to that where the outflow has been detected). The dashed line is the expectation of a SN-driven wind, by assuming $0.0066 \mathrm{SNe}$ per solar mass of newly formed star (Salpeter IMF) a total luminosity for each SN of $10^{51} \mathrm{erg} / \mathrm{s}$ and an efficiency of releasing this luminosity in the ISM to drive a shock of $10 \%$. The solid red line is the expected SFR obtained using the Netzer (2009) relationship between SFR and AGN bolometric luminosity and assuming the average $\dot{E}_{\mathrm{kin}} / L_{\mathrm{bol}}=0.025$ found for molecular winds in Fig. 1 . Right panel: AGN bolometric luminosity as a function of the host galaxy star formation rate. The red, magenta and cyan lines in the right panel are the expected relations based on the SFR- $L_{\mathrm{bol}}$ correlations by Netzer (2009), Hickox et al. $(2014 ; z=0)$ and Hickox et al. $(2014, z=2)$, respectively. Symbols as in Fig. 1.

the Appendix. $v_{\max }$ correlates with the bolometric luminosity for molecular winds, and ionised winds. Considering the two winds together again produces a strong correlation and a log linear slope of $4.6 \pm 1.5$ (see Table 1). For X-ray absorbers the situation is more complex, since they are divided in two broad groups, warm absorbers with lower velocities and UFOs with higher velocities. For UFOs with $v_{\max }>10^{4} \mathrm{~km} \mathrm{~s}^{-1}$ the correlation between AGN bolometric luminosity and maximum velocity is still remarkably strong, with a log linear slope of $3.9 \pm 1.3$ (Table 1), statistically consistent with that of molecular+ionised winds. This means that at each given bolometric luminosity the ratio between UFO maximum velocity and molecular-ionised wind maximum velocity is similar, and equal to $\sim 40-50$. We also report in Fig. 2 the scalings found by Spoon et al. (2013) and Veilleux et al. (2013) for OH outflows in samples of ULIRGs and QSOs at $z<0.3$. Four of the objects in Veilleux et al. (2013) are also part of our sample, see Table B.1.

BALs and the lower velocity X-ray absorbers $v_{\max }<$ $10^{4} \mathrm{~km} \mathrm{~s}^{-1}$ (the so called X-ray warm absorbers), also seem to show a correlation between AGN bolometric luminosity and maximum velocity, with a slope close to the fourth-fifth power, with the warm absorbers present in low luminosity systems and BALs present in high luminosity systems.

The right panel of Fig. 2 shows the wind momentum load (i.e. the wind momentum rate, $\dot{P}_{\mathrm{OF}}=\dot{M}_{\mathrm{OF}} \times v_{\mathrm{max}}$, divided by the AGN radiation momentum rate, $\dot{P}_{\mathrm{AGN}}=L_{\mathrm{bol}} / c$ ) as a function of $v_{\max }$ (see also Stern et al. 2016). The blue solid lines are the expectations for energy conserving winds $\left(\dot{P}_{\mathrm{OF}} / \dot{P}_{\mathrm{AGN}} \approx v_{\mathrm{UFO}} / v_{\mathrm{OF}}\right)$ for the cases of Markarian 231 and IRAS F11119+13257, the only two sources for which both X-ray winds and molecular winds have been detected (Tombesi et al. 2015; Feruglio et al. 2015). Molecular winds have momentum load in the range 3100 , about half have momentum load $>10$, suggesting again that most massive-extended outflows are not momentum conserving but rather energy conserving winds, extended on the host galaxy scales.
Ionised winds have velocities intermediate between molecular winds and X-ray absorbers. The range of their momentum load is wide, from 0.01 to 30 . Most BAL and X-ray winds have $\dot{P}_{\mathrm{OF}} / \dot{P}_{\mathrm{AGN}} \lesssim 1$, suggesting that they are probably momentum conserving, as predicted by the King (2003) model.

\subsection{Wind parameters vs. host galaxy star formation rate}

We now study the correlations between massive, extended winds, i.e. molecular and ionised winds, and the properties of their host galaxies.

Figure 3 shows the outflow kinetic power and AGN bolometric luminosity as a function of SFR in the host galaxy (correlation coefficients given again in Table 1). There is a loose correlation between $\log \left(\dot{E}_{\text {kin }}\right)$ and $\log (\mathrm{SFR})$. It should be kept in mind that the SFR plotted in Fig. 3 is, in most cases, not the instantaneous SFR but rather the conversion from the observed FIR luminosity. The instantaneous SFR can be zero in these systems, and what we are observing is light from stars born hundreds of millions of years before the AGN shutting off and its feedback. This SFR is therefore an upper limit to the on going SFR. Indeed, Davies et al. (2007) found that the on going SFR in the nuclei of Markarian 231 and NGC 1068 is probably very small, because of the small observed $\mathrm{Br} \gamma$ equivalent width within $0.1-0.5 \mathrm{kpc}$ from the active nucleus.

A correlation between $\dot{E}_{\text {kin }}$ and SFR would naturally emerge if winds were supernova (SN) driven. The dashed line in Fig. 3, left panel, is the expectation for SN-driven winds, by assuming $0.0066 \mathrm{SNe}$ per solar mass of newly formed star (Salpeter IMF), a total luminosity for each SN of $10^{51} \mathrm{erg} / \mathrm{s}$, and a $10 \%$ efficiency in releasing this luminosity into the ISM to drive a shock. The SN rate per solar mass is 0.0032 and $0.0083 M_{\odot}^{-1}$ for a Scalo and Chabrier IMF, respectively (Somerville \& Primack 1999; Dutton $\&$ van der Bosh 2009). Therefore, SNe do not seem powerful or numerous enough to drive most observed winds. 
Table 1. Correlations of wind parameters with AGN bolometric luminosity and host galaxies SFR.

\begin{tabular}{|c|c|c|c|c|}
\hline Correlation & Slope & Spearman Rank (SR) & d.o.f. & Null hypothesis probability \\
\hline \multicolumn{5}{|c|}{ Molecular outflows } \\
\hline$\dot{M}_{\mathrm{OF}}$ vs. $L_{\mathrm{bol}}$ & $0.76 \pm 0.06$ & 0.86 & 15 & $<10^{-5}$ \\
\hline$\dot{E}_{\text {kin }}$ vs. $L_{\text {bol }}$ & $1.29 \pm 0.08$ & 0.87 & 15 & $<10^{-5}$ \\
\hline$v_{\max }$ vs. $L_{\text {bol }}$ & $3.4 \pm 0.5$ & 0.80 & 15 & $<10^{-5}$ \\
\hline$L_{\text {bol }}$ vs. SFR & $0.9 \pm 0.3$ & 0.45 & 15 & $3.6 \%$ \\
\hline$\dot{E_{\text {kin }}}$ vs. SFR & $1.2 \pm 0.4$ & 0.33 & 15 & $4.3 \%$ \\
\hline \multicolumn{5}{|c|}{ Ionised outflows } \\
\hline$\dot{M}_{\mathrm{OF}}$ vs. $L_{\mathrm{bol}}$ & $1.29 \pm 0.38$ & 0.72 & 49 & $<10^{-5}$ \\
\hline$\dot{E}_{\text {kin }}$ vs. $L_{\text {bol }}$ & $1.48 \pm 0.37$ & 0.75 & 49 & $<10^{-5}$ \\
\hline $\mathrm{v}_{\max }$ vs. $L_{\mathrm{bol}}$ & $6.1 \pm 4.4$ & 0.34 & 49 & $0.7 \%$ \\
\hline$L_{\text {bol }}$ Vs. SFR & $2.2 \pm 1.6$ & 0.34 & 34 & $2 \%$ \\
\hline$\dot{E}_{\text {kin }}$ vs. SFR & $4.9 \pm 4.4$ & 0.19 & 34 & $13 \%$ \\
\hline \multicolumn{5}{|c|}{$\mathrm{X}$-ray outflows } \\
\hline$\dot{M}_{\mathrm{OF}}$ vs. $L_{\mathrm{bol}}$ & $1.12 \pm 0.16$ & 0.75 & 27 & $<10^{-5}$ \\
\hline$\dot{E}_{\text {kin }}$ vs. $L_{\text {bol }}$ & $2.0 \pm 0.4$ & 0.69 & 27 & $<10^{-5}$ \\
\hline \multicolumn{5}{|c|}{ Molecular + Ionised outflows } \\
\hline $\mathrm{v}_{\max }$ vs. $L_{\mathrm{bol}}$ & $4.6 \pm 1.8$ & 0.57 & 67 & $<10^{-5}$ \\
\hline$L_{\text {bol }}$ vs. SFR & $1.5 \pm 0.6$ & 0.44 & 52 & $4 \times 10^{-4}$ \\
\hline$\dot{E}_{\text {kin }}$ vs. SFR & $2.1 \pm 1.4$ & 0.28 & 52 & $2 \%$ \\
\hline \multicolumn{5}{|c|}{ Ultra fast outflows } \\
\hline$\dot{M}_{\mathrm{OF}}$ vs. $L_{\mathrm{bol}}$ & $1.13 \pm 0.11$ & 0.90 & 18 & $<10^{-5}$ \\
\hline$\dot{E}_{\text {kin }}$ vs. $L_{\text {bol }}$ & $1.44 \pm 0.11$ & 0.87 & 18 & $<10^{-5}$ \\
\hline$v_{\max }$ vs. $L_{\mathrm{bol}}$ & $3.9 \pm 1.4$ & 0.46 & 18 & $0.4 \%$ \\
\hline
\end{tabular}

If the winds are AGN driven, a correlation between $\dot{E}_{\mathrm{kin}}$ and SFR would actually be expected because of the correlation between $L_{\mathrm{bol}}$ and SFR (Fig. 3 right panel). Several authors published correlations between AGN luminosity and SFR, whose scatter is large, in particular at low AGN luminosities (e.g. Shao et al. 2010; Rosario et al. 2012; Mullaney et al. 2012; Hickox et al. 2014; Rodighiero et al. 2015). As an example, Mullaney et al. (2015) find that the distribution of the offset from the main sequence $S F R / S F R_{\mathrm{MS}}$ of X-ray selected AGN in CDFS follows a log-normal distribution with $\sigma \sim 0.6 \mathrm{dex}$, nearly independent on redshift. We plot in Fig. 3, right panel, the expected SFR based on the SFR $-L_{\text {bol }}$ correlations by Netzer (2009), Hickox et al. (2014), $z=0$, and Hickox et al. (2014), $z=2$. It should be kept in mind that these correlations concern the average SFR. It should also be kept in mind that these correlations are probably driven by scaling laws. Larger systems are more likely to have higher luminosities, more powerful outflows and larger SFRs. What really matters is the size of the system (also see Mancuso et al. 2016).

Figure 4 shows the mass-loading factor, $\eta=\dot{M}_{\mathrm{OF}} / \mathrm{SFR}$, as a function of AGN bolometric luminosity and stellar mass. The mass-loading factor of molecular winds is $>1$ in most cases, and $>10$ in about half the cases. The median mass-loading factor of ionised winds is $\approx 1$, with a rather large distribution between 0.001 and 100. $\eta$ is not correlated with the AGN bolometric luminosity while it is weakly anticorrelated with stellar mass. The AGN driven wind mass-loading factors are systematically larger than those of starburst driven winds in local star-forming galaxies (Heckman et al. 2015, yellow band in Fig. 4).

\subsection{Molecular gas fractions and depletion timescales of AGN with massive winds}

For the sample of AGN with CO measurements we compute the depletion timescale (i.e. the time needed to convert all molecular gas into stars at the current star formation rate), $t_{\mathrm{dep}}(S F)=$ $M_{\mathrm{gas}} / S F R$ ), and the molecular gas fraction (i.e. the ratio of the molecular gas mass to stellar mass $\left.f_{\text {gas }}=M_{\text {gas }} / M_{*}\right)$. We can then compare the distributions of $t_{\mathrm{dep}}$ and $f_{\text {gas }}$ to the corresponding Genzel et al. (2015) scaling relations. We use the equations in Whitaker et al. (2012) and Genzel et al. (2015) to compute the specific SFR of the galaxy main sequence (MS) as a function of redshift and stellar mass, $s S F R_{\mathrm{MS}}$.

Figure 5 shows the offset from the MS, $\log \left(s S F R / s S F R_{\mathrm{MS}}\right)$, as a function of the stellar mass for the samples of ionised and molecular winds. This distribution is the result of assembling an heterogeneous sample, with different selection criteria, and shows how much the present sample is biased toward starbursts systems. In fact, the first molecular outflows were found in local starburst galaxies hosting an AGN (Fischer et al. 2010; Feruglio et al. 2010; Sturm et al. 2011). Conversely, ionised outflows at $z \sim 2$ are from samples of SMGs or QSOs. It is important to consider these different selection criteria and distributions of source samples with respect to the MS for the following discussion.

Following Genzel et al. (2015), Figs. 6 and 7 show the depletion timescale and the molecular gas fraction as a function of the the offset from the MS and stellar mass, after normalisation for the trends with redshifts and offset from the MS (i.e. the functions $f_{1}(z)$ and $g_{1}\left(s S F R / s S F R_{\mathrm{MS}}\right)$ and $f_{2}(z)$ and $g_{2}\left(s S F R / s S F R_{\mathrm{MS}}\right)$, Genzel et al. 2015). We find that the depletion timescale, normalised for the offset from the MS and the trend with the redshift is between -1 and -0.5 dex shorter than the average for $M_{*}<10.5$, further reducing at higher stellar masses (the only point close to average is the $z=6.4$ QSO J1148+5251, which has the most uncertain estimate of both stellar mass and SFR). The normalised gas mass is also systematically smaller than the average found by Genzel et al. (2015; a factor of $\sim 10$ at $M_{*}=10.5$ ). The normalised gas mass reduces at high stellar masses with a slope similar to that of the average ( -0.5, Genzel et al. 2015).

Brusa et al. (2015b) proposed that feedback due to strong winds in massive AGN host galaxies may be the cause of the shorter depletion timescales and smaller molecular gas measured in a $z 1.5$ obscured QSO (XID2028, green triangle) and this may 

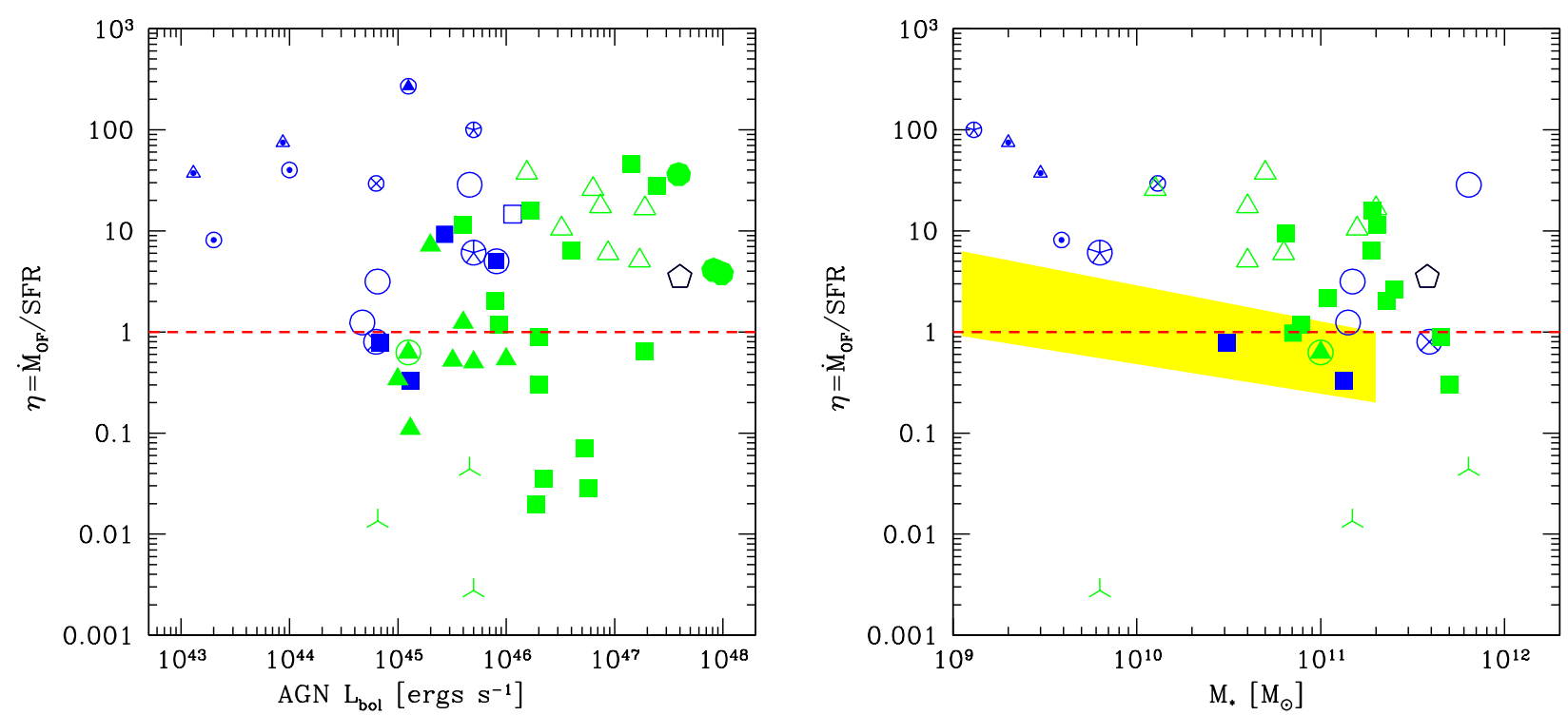

Fig. 4. Mass-loading factor $\eta=\dot{M}_{\mathrm{OF}} / \mathrm{SFR}$ as a function of AGN bolometric luminosity left panel), host galaxy stellar mass right panel. The yellow band in the right panel is the range found by Heckman et al. (2015) for starburst driven galactic winds in a sample of local star-forming galaxies. Symbols as in Fig. 1.

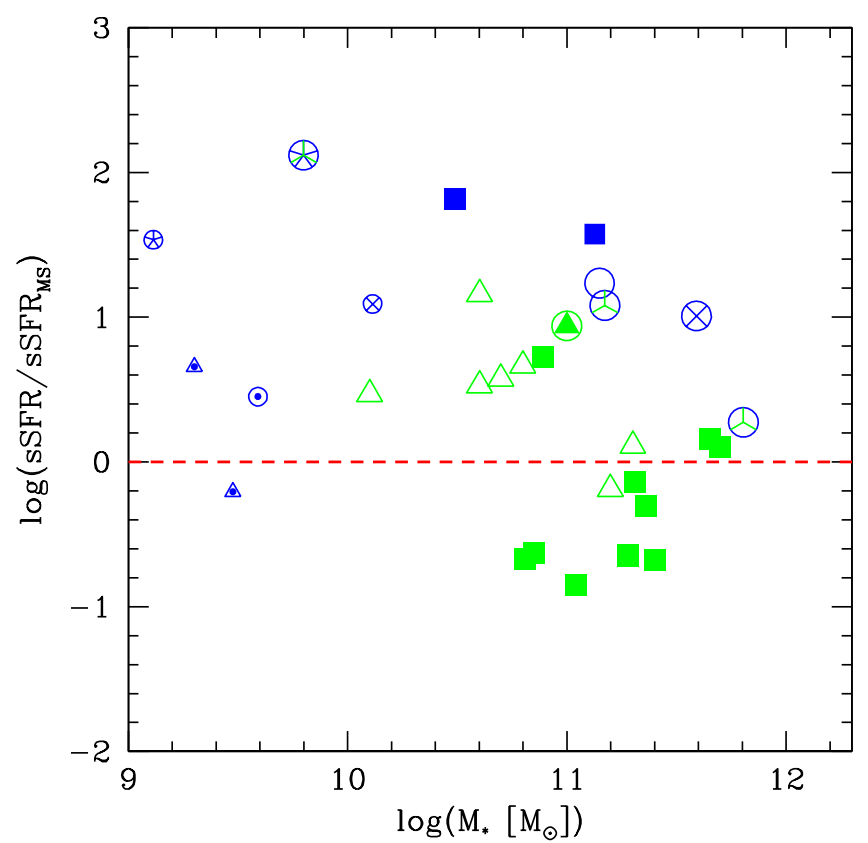

Fig. 5. Offset from the galaxy main sequence ( $\left.\mathrm{sSFR} / \mathrm{sSFR}_{\mathrm{MS}}\right)$ as a function of the AGN host galaxy stellar mass. Symbols as in Fig. 1.

also be the case for the galaxies in our sample. However, we note that part of the offset of galaxies hosting powerful molecular winds from average star-forming galaxies may be due to the adopted conversion factor from $L^{\prime}(\mathrm{CO})$ to $M_{\text {gas }}$ (we adopted $\alpha_{\mathrm{CO}}=0.8$ for our sample, mostly made by LIRGs and ULIRGs, while Genzel et al. 2015 use a complex conversion function, that takes into account metallicity and density-temperature dependence). This may account for up to a factor of $\sim 3-4$ in $t_{\mathrm{dep}}$ and $f_{\text {gas }}$. Even taking into account this correction, most points in Figs. 6 and 7 would fall short of the Genzel et al. (2015) average values, in particular at high stellar masses. It should also be considered that the Genzel et al. (2015) averages themselves (i.e. the average depletion timescales and gas fraction after the subtraction of the trends with redshift and offset from the main sequence), may well be affected by uncertainties. For example, the Sargent et al. (2014) parameterisation results in a depletion timescale a factor of $\sim 2$ shorter than the Genzel et al. (2015) one for galaxies above the MS.

Figure 8 shows the star formation against the outflow depletion timescales, $t_{\mathrm{dep}}(\mathrm{OF})=M_{\text {gas }} / \dot{M}_{\mathrm{OF}}$. At face value, in most systems, and in particular the six galaxy nuclei, outflows are powerful enough to deplete the galaxy molecular gas reservoir in a timescale shorter than that needed to exhaust it by forming stars at the measured rate (red dashed line). This assumes that the molecular winds are not blocked at some distance and do not dissolve out. Pressure-confined molecular clouds may, however, dissolve out as the wind expands, and $\mathrm{CO}$ may be efficiently photo-dissociated by the UV radiation, since self shielding will be strongly reduced at low densities. The best studied molecular wind so far (Markarian 231, Feruglio et al. 2015) has a size of $\sim 1 \mathrm{kpc}$. At this distance the mass in outflow strongly reduces, while its velocity remains nearly constant, suggesting that a large part of the molecular gas leaves the flow during its expansion. This molecular gas may rain back onto the nucleus or the disk, replenishing the gas reservoirs.

\section{AGN winds in a cosmological framework}

We now attempt to put the results from the previous section in the cosmological evolution framework. This will enable us to assess the relative importance of AGN driven winds on the average cosmological star formation, accounting for the fraction of galaxies which are caught in the AGN phase. This fraction can be as low as $1 \%$ in the local Universe, and up to $30 \%$ at $z=2$ (Brusa et al. 2009; Fiore et al. 2012; Bongiorno et al. 2012). We first summarise the results on the evolution of AGN and galaxies luminosity densities, we then link SMBH accretion to star formation, and finally estimate the AGN wind mass loading factor density as a function of the cosmic time. 

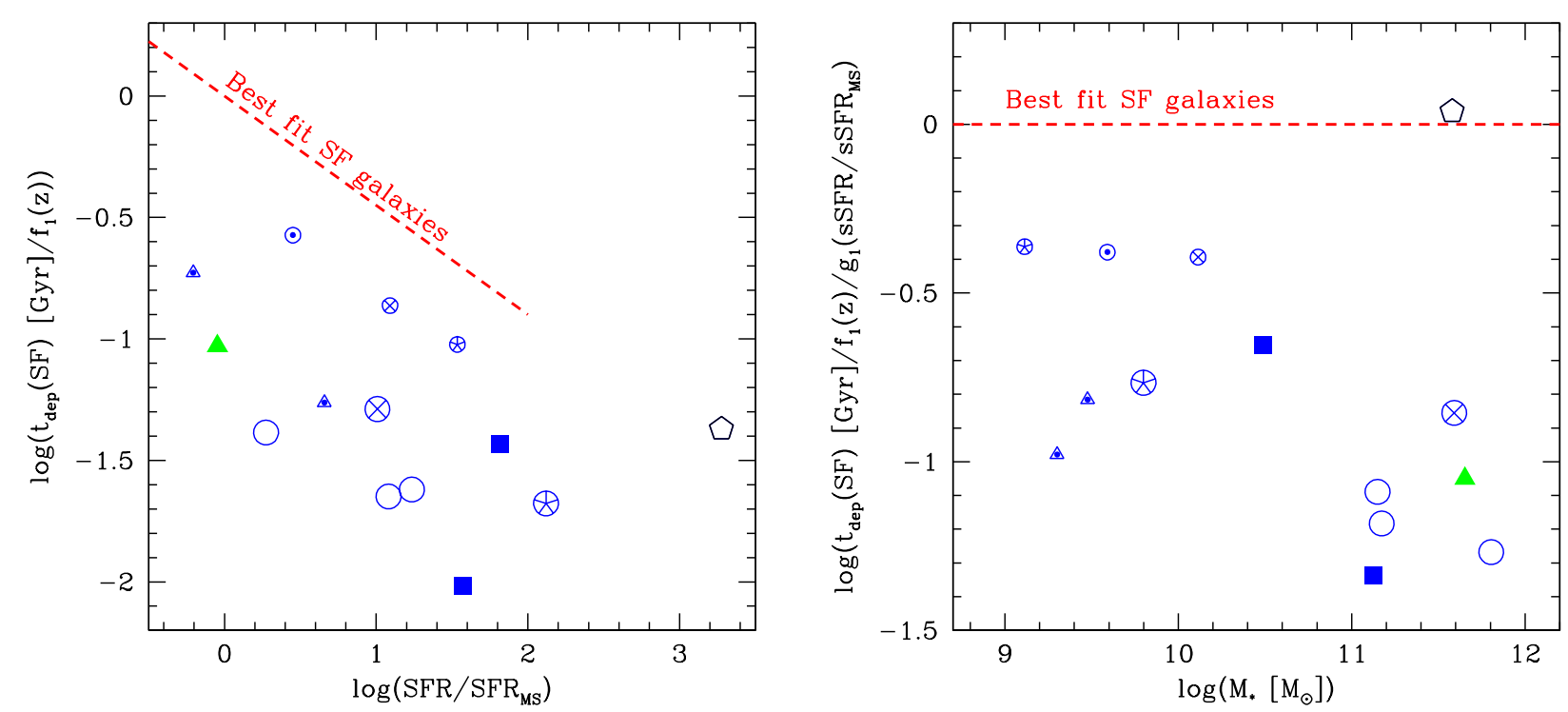

Fig. 6. Left panel: depletion timescale, $t_{\mathrm{dep}}(S F)=M_{\mathrm{gas}} / S F R$, as a function of the offset from the main sequence, after normalisation to the midline of main sequence at each redshift, by removing the redshift dependences with the fitting functions $f_{1}(z)$ in Table 3 of Genzel et al. (2015) corresponding to $\mathrm{CO}$ data, global distribution. Right panel: depletion timescale as a function of the galaxy stellar mass, after normalisation to the mid-line of main sequence, by removing the specific star formation rate dependence with the fitting function $\mathrm{g} 1\left(\mathrm{sSFR} / \mathrm{s} S F R\left(m s, z, M_{*}\right)\right.$ in Table 3 of Genzel et al. (2015) for CO data, global distribution. Symbols as in Fig. 1. The black pentagon marks J1148+5251 at $z=6.4$. The green triangle marks the QSO XID2028 with a detected ionised wind and measured molecular gas mass (Cresci et al. 2015; Brusa et al. 2015b). The red, dashed lines are the best linear fits to the log-log distributions of $500 \mathrm{CO}$ star-forming galaxies in Genzel et al. (2015).
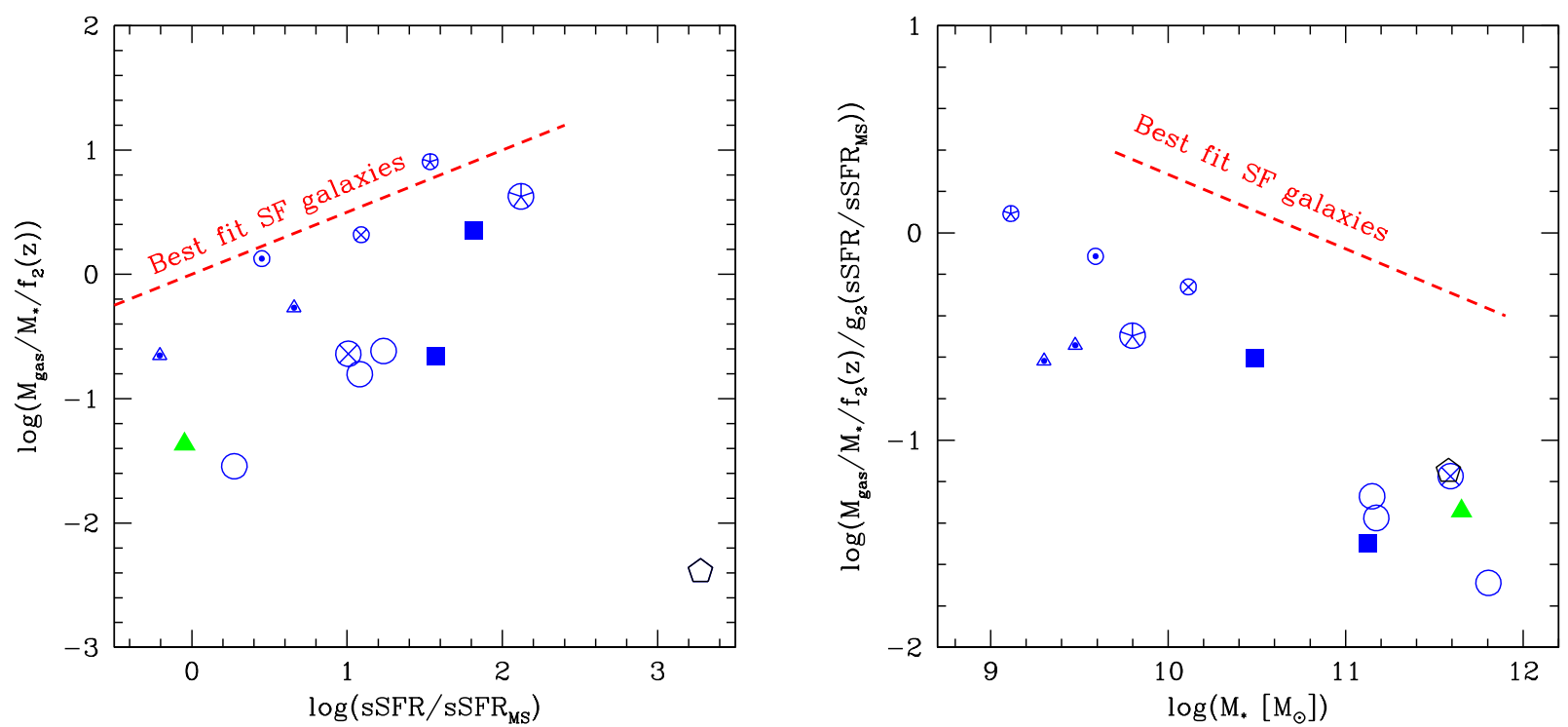

Fig. 7. Left panel: molecular gas fraction $f_{\text {gas }}=M_{\text {gas }} / M_{*}$ as a function of the offset from the main sequence, after normalisation to the midline of main sequence at each redshift, by removing the redshift dependences with the fitting functions $f_{2}(z)$ in Table 4 of Genzel et al. (2015) corresponding to $\mathrm{CO}$ data, global distribution. Right panel: molecular gas fraction as a function of the galaxy stellar mass, after normalisation to the mid-line of main sequence, by removing the specific star formation rate dependence with the fitting function $\mathrm{g} 2\left(s S F R / s S F R\left(m s, z, M_{*}\right)\right.$ in Table 4 of Genzel et al. (2015) for CO data, global distribution. The black pentagon marks J1148+5251 at $z=6.4$. The green triangle marks the QSO XID2028 with a detected ionised outflow and measured molecular gas mass (Cresci et al. 2015; Brusa et al. 2015b). The red, dashed lines mark the average correlations found by Genzel et al. 2015.

\subsection{The evolution of the AGN luminosity density}

We plot in Fig. 9 the evolution of the X-ray 2-10 keV AGN luminosity density for different AGN luminosities. AGN as faint as $L(2-10 \mathrm{keV})=10^{42} \mathrm{erg} / \mathrm{s}$ can be detected by Chandra in ultradeep surveys, even up to $z=2.5-3$. Brighter AGN with $L(2-$ $10)=>10^{43} \mathrm{erg} / \mathrm{s}$ are observable up to $z=6$. For this reason, we provide two distinct plots, one including $10^{42} \mathrm{erg} / \mathrm{s}$ AGN up to $z=2.5$ and another including $L(2-10)=>10^{43} \mathrm{erg} / \mathrm{s}$ up to $z=6$. The shaded areas account for the uncertainties. AGN samples at $z<3$ include today several thousands objects, resulting into small statistical errors on the luminosity functions up to this redshift. In particular, the statistical error is smaller than the systematic error due to the different assumptions that authors make to account for selection effects. At $z<3$ the shaded areas bracket the determinations of La Franca et al. (2005), 


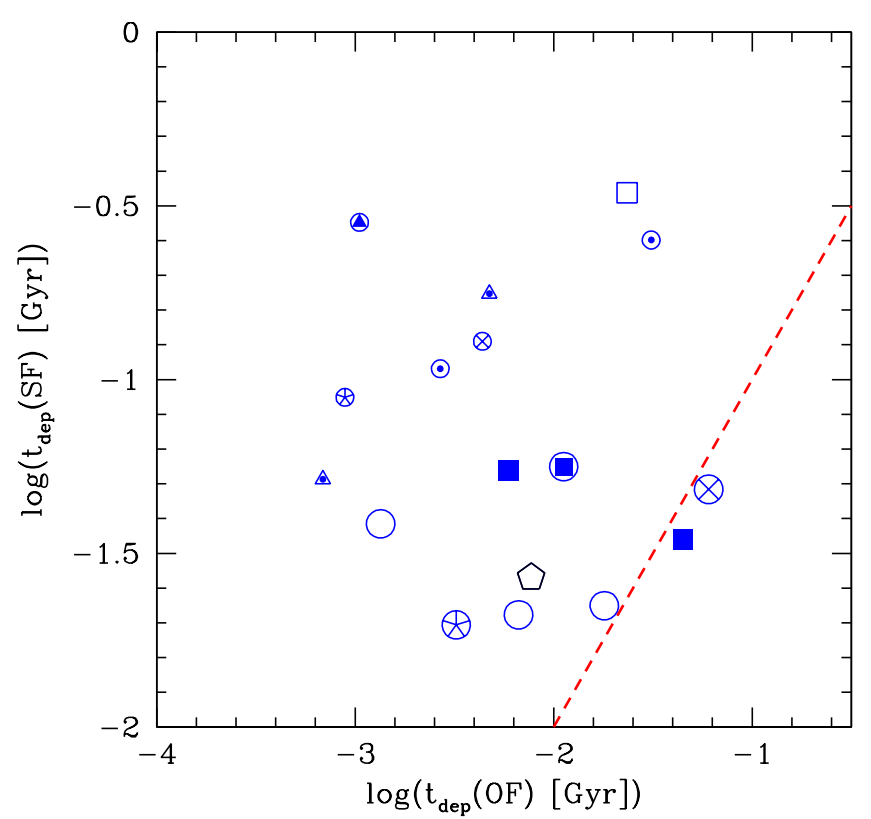

Fig. 8. SFR depletion timescale, $t_{\text {dep }}(S F)=M_{\text {gas }} / S F R$, as a function of the wind depletion timescale, $t_{\mathrm{dep}}(\mathrm{OF})=M_{\mathrm{gas}} / \dot{M}_{\mathrm{OF}}$. The red dashed line is the depletion timescale due to star formation at the measured rate.

Ebrero et al. (2009), Aird et al. (2010), and Ueda et al. (2014). Conversely, X-ray selected AGN samples at $z>3$ are still relatively small, including hundred objects at $z=3-4$, a few dozen objects at $z>4$, and a few at $z>6$. Optically selected AGN at $z>6$ are relatively rare too, with only $\sim$ a few dozen luminous QSOs at $z>6$ known so far. As a consequence, the main error source on the AGN luminosity functions at the low-medium luminosities sampled by X-ray surveys at $z>3$, and by optical surveys at $z>6$, is the statistical error. At $z>3$ we used the Fiore et al. (2012), Ueda et al. (2014), Georgakakis et al. (2015), Aird et al. (2015), Kalfountzou et al. (2014), Vito et al. (2014), Marchesi et al. (2016), and Puccetti et al. (in prep.) AGN luminosity functions. The shaded areas account for both statistical and systematic errors. The left panel of Fig. 9 clearly shows the downsizing of AGN X-ray luminosity density, with AGN of X-ray luminosity $10^{43}-10^{44} \mathrm{erg} / \mathrm{s}$ peaking at $z=1$, and AGN of X-ray luminosity $>10^{45} \mathrm{erg} / \mathrm{s}$ peaking at $z=z 2$. The total AGN X-ray luminosity density peaks at $z=1-2$ (right panel). The right panel of Fig. 9 also shows the galaxy UV luminosity density, scaled by a factor $10^{3}$ (from Bouwens et al. 2011, 2015; Santini et al. 2009; Gruppioni et al. 2015; Madau \& Dickinson 2014). Note that the total galaxy UV luminosity density peaks at $z=2-3$, a redshift higher than that of the peak of the AGN $\mathrm{X}$-ray luminosity density.

While the galaxy UV luminosity is linearly correlated with the SFR, the X-ray luminosity is not a good proxy of the AGN bolometric luminosity, and thus of the SMBH gas accretion rate. The relationship between X-ray luminosity and the AGN bolometric luminosity is complex and may depend on several parameters. Several authors suggested a polynomial scaling between $\log L_{\mathrm{X}}$ and $\log L_{\mathrm{bol}}$ (Marconi et al. 2004; Hopkins et al. 2006). Others suggest a scaling with the Eddington ratio (Vasudevan \& Fabian 2007; Jin et al. 2012). As an example, we adopt the Marconi et al. (2004) scaling to calculate the evolution of the AGN bolometric luminosity density given in Table 2 and plotted in Fig. 10 (we integrate the X-ray AGN luminosity functions in the luminosity range $10^{42}-10^{45} \mathrm{erg} / \mathrm{s}$ ). This is compared with the similar determination of Aird et al. (2015) and with the
Table 2. AGN bolometric luminosity density evolution.

\begin{tabular}{lcc}
\hline \hline Redshift & $\log L(\min )$ & $\log L(\max )$ \\
\hline 0.1 & 40.1 & 40.4 \\
0.25 & 40.6 & 40.7 \\
0.5 & 40.8 & 41.0 \\
1 & 41.0 & 41.3 \\
1.5 & 41.2 & 41.5 \\
2 & 41.3 & 41.6 \\
2.5 & 41.4 & 41.5 \\
3.25 & 41.3 & 41.3 \\
3.75 & 40.9 & 41.1 \\
4.5 & 40.5 & 40.9 \\
5.5 & 40.2 & 40.7 \\
6.0 & 40.0 & 40.6 \\
\hline
\end{tabular}

evolution of the UV luminosity density. Our determination of the AGN bolometric luminosity density based on a compilation from the papers quoted above falls short by up to a factor $30 \%$ from the Aird et al. (2015) determination (total AGN luminosity density, included the contribution of Compton thick AGN) at $z \lesssim 3$. Above this redshift it is consistent with the Aird et al. (2015) determination within the, rather large, uncertainties. The AGN bolometric luminosity density is $\sim 10$ times smaller than the UV luminosity density at all redshift. The shape of the AGN bolometric and UV luminosity density are similar, both peaking at $z=1.5-2.5$. Differences are smaller than the systematic differences between different determinations of AGN bolometric (compilation in this work vs. Aird et al. 2015) and UV (compilation in this work vs. Madau \& Dickinson 2014). We remark here that these results are obtained using the Marconi et al. (2004) bolometric correction to convert X-ray to bolometric luminosity, while Aird et al. (2015) used the correction provided by Hopkins et al. (2006). Adopting other scalings, for example assuming a more complex relationship between bolometric luminosity, SMBH mass and Eddington ratio, would produce somewhat different results. We further investigate this issue in the next sections.

\subsection{SMBH accretion and star formation}

A complementary approach to compare SMBH accretion and star formation is to self-consistently evolve the SMBH mass function via the continuity equation (Cavaliere et al. 1971; Small \& Blandford 1992):

$\frac{\partial n_{\mathrm{BH}}}{\partial t}\left(M_{\mathrm{BH}}, t\right)=-\frac{\partial\left(\left\langle\dot{M}_{\mathrm{BH}}\right\rangle n_{\mathrm{BH}}\left(M_{\mathrm{BH}}, t\right)\right)}{\partial M_{\mathrm{BH}}}$

$n_{\mathrm{BH}}\left(M_{\mathrm{BH}}, t\right)$ is the number of SMBHs of mass $M_{\mathrm{bh}}$ at time $t$ and $\left\langle\dot{M}_{\mathrm{BH}}\left(M_{\mathrm{BH}}, t\right)\right\rangle$ is the mean accretion rate, averaged over the active and inactive populations, of all SMBHs of mass $M_{\mathrm{bh}}$ at time $t$. While Eq. (1) neglects any contribution from SMBH mergers, the latter process does not impact the mean accretion rate but it mainly alters the redistribution of the mass function (Shankar et al. 2009). The average growth rate of all SMBHs can be computed by convolving the probability to radiate at a given fraction $\lambda=L / L_{\text {Edd }}$ of the Eddington luminosity $P\left(\lambda \mid M_{\mathrm{BH}}, z\right)$, and the overall probability, or "duty cycle", to be active $U\left(M_{\mathrm{BH}}, z\right)$

$\left\langle\dot{M}_{\mathrm{BH}}\right\rangle=\int \mathrm{d} \log \lambda P\left(\lambda \mid M_{\mathrm{BH}}, z\right) \lambda U\left(M_{\mathrm{BH}}, z\right) \frac{M_{\mathrm{BH}}}{t_{\mathrm{s}}}$, 

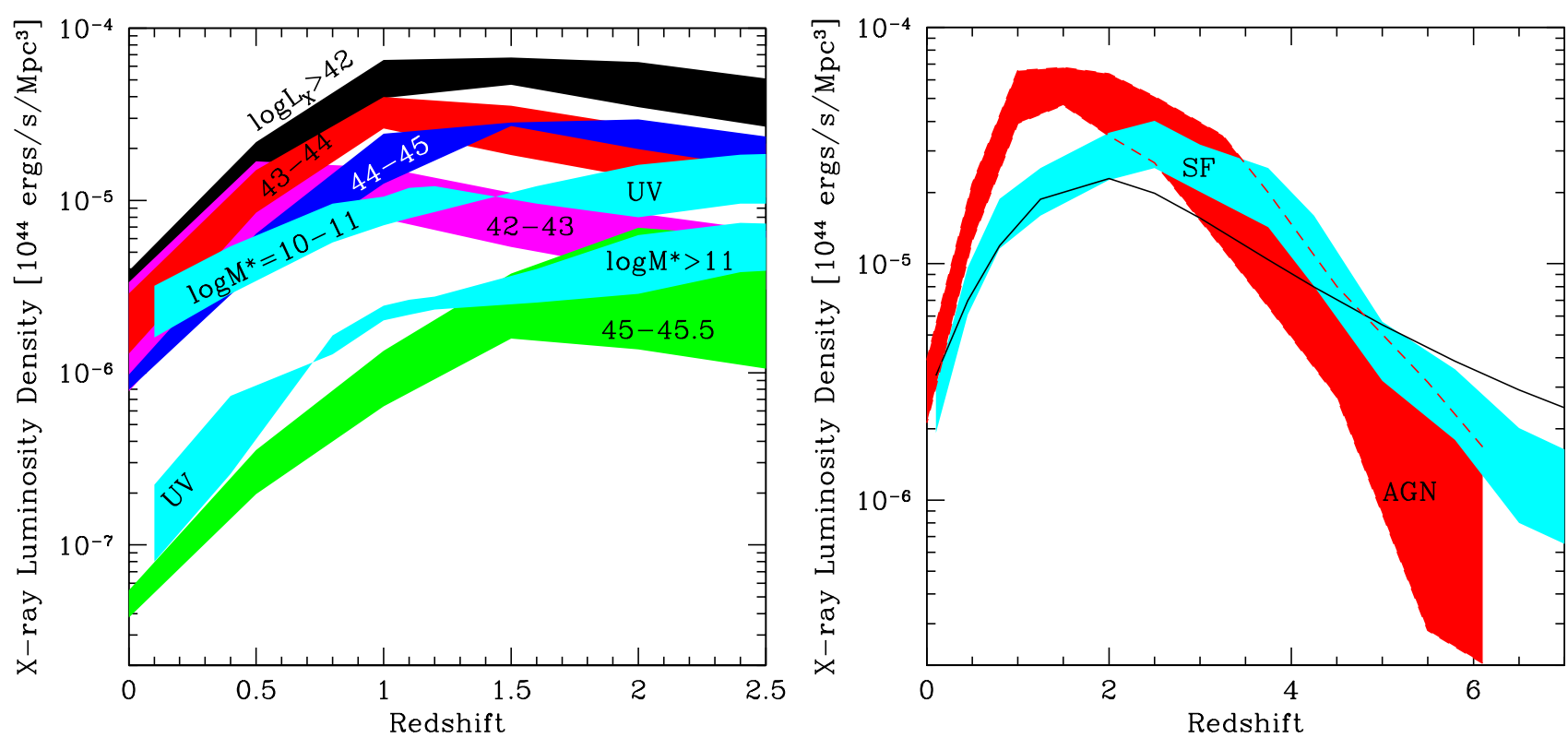

Fig. 9. Evolution of the X-ray 2-10 keV AGN luminosity density for different AGN luminosities. Left panel: AGN luminosity density for AGN, split in ranges of 2-10 keV luminosity, as labelled. Cyan bands show the SF luminosity density as estimated by Santini et al. (2009) and Gruppioni et al. (2015), divided by a factor of 1000 for plotting purpose, for galaxies with $\log M_{*}=10-11$ and $\log M_{*}>11$. Right panel: total AGN luminosity density for AGN with $L(2-10)=>10^{43} \mathrm{erg} / \mathrm{s}$ (red band). SF luminosity density as estimated by Santini et al. (2009), Gruppioni et al. (2015), and Bouwens et al. $(2011,2015)$ and divided by a factor 1000 for plotting purposes (cyan band). Average SF luminosity density as estimated by Madau \& Dickinson (2014; black solid line).

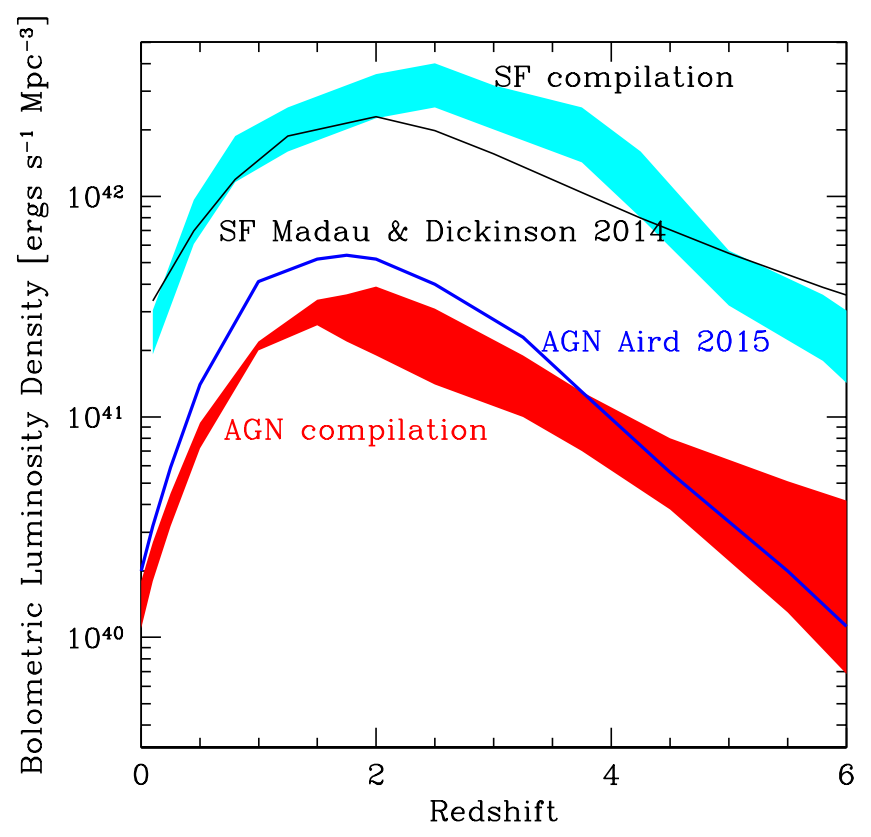

Fig. 10. Evolution of the AGN bolometric luminosity density: the red band has been computed from a compilation of X-ray luminosity functions integrated in the range $\log L_{X}=42-45$, se text for details, and assuming the Marconi et al. (2004) bolometric correction; the blue solid line is the Aird et al. (2015) determination. Fort the UV luminosity density the cyan band is the average of a compilation from Santini et al (2009), Gruppioni et al. (2015), Bouwens et al. (2011, 2015). The black solid line is the Madau \& Dickinson (2014) determination.

where $t_{s}$ is the AGN Salpeter timescale and the integral extends over all allowed values of $\lambda$. The input Eddington ratio distributions are motivated by a variety of independent observational probes, while the duty cycle is self-consistently re-computed at each time $t$ from the ratio between the AGN luminosity function and SMBH mass function at the previous time step (the full methodology and numerical details can be found in, e.g. Shankar et al. 2013, and references therein).

It has been already emphasised in the literature that the average SMBH accretion rate density has a redshift dependence morphologically similar to the cosmological SFR density (Marconi et al. 2004; Merloni et al. 2004; Silverman et al. 2008; Zheng et al. 2009; Shankar et al. 2009). We provide in Fig. 11 our estimate of the ratio between SMBH growth and SFR density. We first extract $\left\langle\dot{M}_{\mathrm{BH}}\left(M_{\mathrm{BH}}, z\right)\right\rangle$ from the continuity equation models of Shankar et al. (2013), and bin it in the ranges $7<$ $\log M_{\mathrm{bh}} / M_{\odot}<8,8<\log M_{\mathrm{bh}} / M_{\odot}<9$, and $\log M_{\mathrm{bh}} / M_{\odot}>9$, which implies integrating it over the appropriate mass range of active SMBH mass function at all epochs. We thus convert an accretion rate, measured in $M_{\odot} \mathrm{yr}^{-1}$ to a SMBH accretion rate density, measured in $M_{\odot} \mathrm{yr}^{-1} \mathrm{Mpc}^{-3}$. We then divide the accretion rate density for the average SFR density. Finally, we take the SFR densities in the stellar mass ranges $10<\log M_{\text {star }} / M_{\odot}<11$ and $\log M_{\text {star }} / M_{\odot}>11$ and relate then to the accretion rate densities from SMBH of masses $7<\log M_{\mathrm{bh}} / M_{\odot}<8$ and $\log M_{\mathrm{bh}} / M_{\odot}>8$, respectively. This allows us to infer an approximate mass-dependent correlation between $\left\langle\dot{M}_{\mathrm{BH}}\left(M_{\mathrm{BH}}, z\right)\right\rangle$ and SFR density. All models predict, on average, a nearly constant ratio in time of the SMBH mean accretion and SFR (red shaded area). The top panels corresponds to constant Eddington ratio distribution at all redshifts and constant radiative efficiency $\epsilon=0.06$ (left) and $\epsilon=0.2$ (right). Note that the average ratio between the SMBH accretion rate density and SFR density is about $3 \times 10^{-4}$ and $10^{-4}$ for $\epsilon=0.06$ and $\epsilon=0.2$, respectively. Note that the latter ratio is consistent with the intrinsic value computed by Shankar et al. (2016) for the same $\epsilon$. At all redshifts the ratio is higher for massive galaxies. The peak ratio of less massive galaxies is at $z=0.5-1$ (which is also the redshift at which the density of low luminosity AGN $\left(L_{2-10 \mathrm{keV}} \lesssim 10^{44} \mathrm{erg} / \mathrm{s}\right)$ peak). 

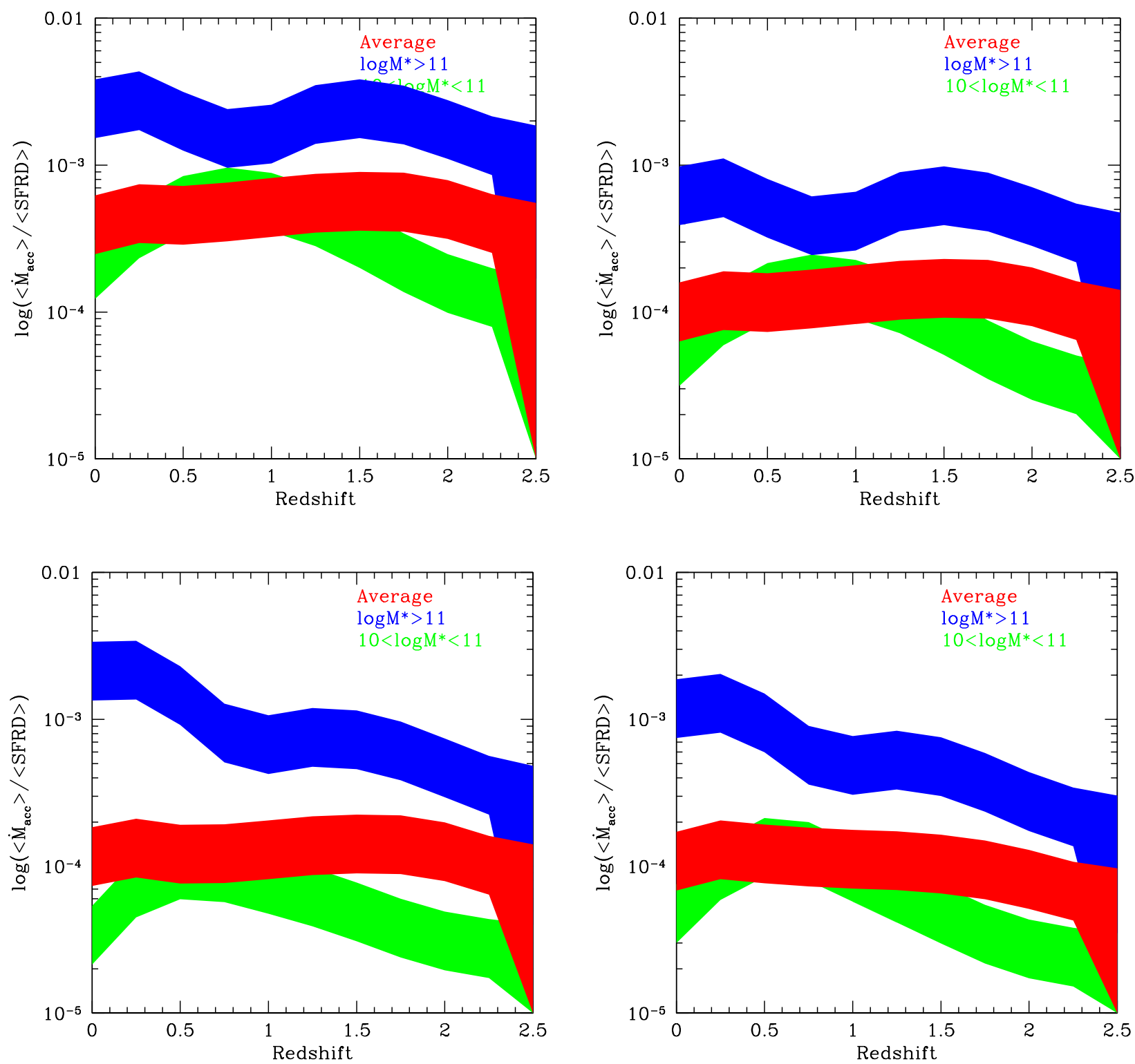

Fig. 11. Ratio between the SMBH accretion rate and SFR densities as a function of the redshift. Upper-left panel: constant radiative efficiency $\epsilon=0.06$ and constant Eddington ratio distribution at all redshifts. Upper-right panel: constant radiative efficiency $\epsilon=0.2$ and constant Eddington ratio distribution at all redshifts. Lower-left panel: an evolving Eddington ratio (e.g. 15 in Shankar et al. 2013), $\epsilon=0.2$. Lower-right panel: mass-dependent radiative efficiency $\epsilon$ from 0.05 to 0.4 linearly with BH black hole mass at all redshifts and evolving Eddington ratio.

Similar conclusions apply to the other models analysed: evolving Eddington ratio (lower-left panel), and mass-dependent radiative efficiency (lower-right panel). Interestingly, the strong, apparent co-evolution between accreting SMBHs and galaxies appears to break down for the least massive galaxies, while the most massive galaxies tend to align with a ratio of $5-10 \times 10^{-4}$ or higher.

\subsection{The evolution of the AGN wind mass-loading factor}

The remarkable correlation between the AGN bolometric luminosity, $L_{\mathrm{bol}}$, AGN wind mass outflow rate, $\dot{M}_{\mathrm{OF}}$, and kinetic power, $\dot{E}_{\text {kin }}$ (see Fig. 1 and Sect. 2) suggests that the AGN bolometric luminosity density can be converted to a density of wind mass outflow rate and kinetic power. This can then be divided by the SFR density to compute an "average" mass-loading factor as a function of the redshift (under the assumption that:

$\left.\langle\eta\rangle=\left\langle\dot{M}_{\mathrm{OF}} D / S F R D\right\rangle \sim\left\langle\dot{M}_{\mathrm{OF}} D\right\rangle /\langle S F R D\rangle\right)$.

To this purpose, we converted the AGN bolometric luminosity density into a density of the AGN mass outflow rate using Monte Carlo realisations. More in detail, we first randomly chose a bolometric luminosity following the luminosity function distribution in each given redshift bin, and then convert it into mass outflow rate assuming $\dot{M}_{\mathrm{OF}} \propto L_{\text {bol }}^{0.76}$ (baseline scaling), and normalisation consistent with the findings for molecular winds in Sect. 2 (we used the scaling $\log \dot{M}_{\mathrm{OF}}=0.76 * \log L_{\mathrm{bol}}-32$, dashed line Fig. 1 left panel). We remark that this scaling refers to a biased sample of local AGN, and we assume that the same scaling holds at all redshifts. To study how much our conclusions depend on the exact form of the scaling, we calculated the mass outflow rate densities also adopting two different scalings: a square root scaling between the AGN mass outflow rate and 


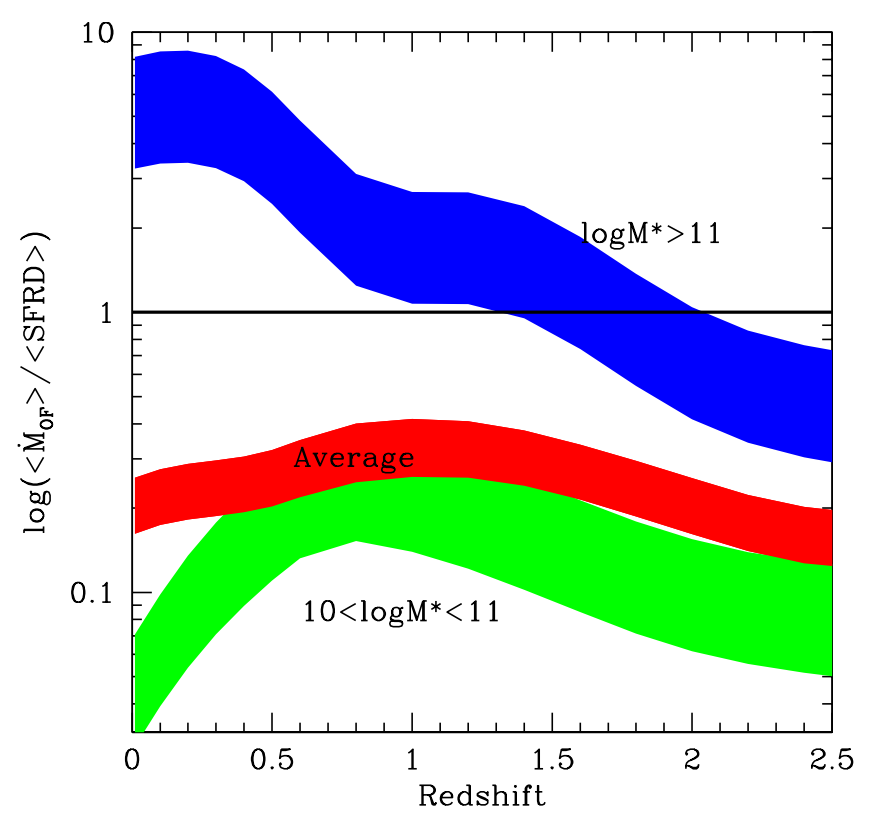

Fig. 12. Average mass-loading factor $\langle\eta\rangle=\left\langle\dot{M}_{\mathrm{OF}} D\right\rangle /\langle S F R D\rangle$ as a function of the redshift. Red = average; blue $=M *>10^{11} M_{\odot}$; green $=10^{10}<M *<10^{11} M_{\odot}$.

the bolometric luminosity and a linear scaling. We first discuss the results obtained with the baseline scaling, and then comment on the differences with respect to the flatter and steeper scalings.

A proper comparison between AGN activity and host galaxy SFR requires at least a rough separation between activity in galaxies of different stellar mass. Santini et al. (2009) and Gruppioni et al. (2015) provides estimates of the SFR for galaxies separated into two mass bins, $\log M_{*}>11$ and $10<\log M_{*}<$ 11. To statistically evaluate the contribution of AGN wind mass outflow rate into these two galaxy mass bins we need to statistically associate to each AGN bolometric luminosity realisation a host galaxy stellar mass. This can be done by associating to the AGN bolometric luminosity a SMBH mass (by assuming a distribution of Eddington ratios), and then converting the $\mathrm{SMBH}$ mass to a stellar mass. This was done using the results briefly presented in the previous section and in Shankar et al. (2013, 2016). In particular, we used the model with $\epsilon=0.06$ and the Eddington ratio distribution given by Eq. (15) of Shankar et al. (2013), and the SMBH mass - galaxy mass correlation given by Eq. (6) of Shankar et al. (2016), assuming an intrinsic dispersion of 0.4 dex. The resulting distributions of AGN wind mass outflow rates (total, and in the two stellar mass bins given above), have been binned to build AGN mass outflow rate density functions. $10^{8}$ realisations have been randomly chosen in each redshift bin. Figure 12, shows the average mass-loading factor, i.e. the ratio between the resulting AGN wind outflow rate density and the average SFR density as a function of the redshift. The average mass-loading factor is between $20 \%$ and $40 \%$ for the average population and peaks at $z \sim 1$. The distributions are quite different when splitting the galaxy population in low mass stellar mass galaxies $\left(10^{10}-10^{11} M_{\odot}\right)$ and high stellar mass galaxies $\left(>10^{11} M_{\odot}\right)$. Small mass galaxies hosting, on average, fainter nuclei with energetically fainter AGN winds, are less affected by AGN winds than larger galaxies, hosting, on average, more luminous nuclei, with more energetic winds. The latter galaxies (stellar masses $>10^{11} M_{\odot}$ ) are, on average, strongly affected by AGN winds at $z \lesssim 2$, where they have $\langle\eta\rangle \gtrsim 1$. The relative importance of AGN winds reduces at $z>2$ also in massive systems, remaining however always higher than that in less massive systems. In this calculation we used the new calibration of the intrinsic SMBH mass-galaxy mass correlation found by Shankar et al. (2016) to split the average mass loading factor into massive and less massive systems. Similar conclusions are obtained using the traditional, biased, correlation.

We calculated the average mass loading factor by assuming a square root and a linear scaling between the wind mass outflow rate and the bolometric luminosity. In the former case the curves shift to slightly lower redshift (peak redshift between $0.5-1$ ), and lower value of the average loading factor (10-30\%), while for the latter case the opposite trend is observed. We also calculate the average mass loading factor by assuming different normalisations of the $\dot{M}_{\mathrm{OF}}-L_{\mathrm{bol}}$ scaling. To bring the average mass loading factor to $\sim 1$ would require a normalisation $\sim 3$ times higher than the dashed line in Fig. 1, left panel, completely inconsistent with the present data. Changing the normalisation within its statistical error does not change significantly the conclusions described above.

\section{Discussion}

\subsection{AGN wind scaling relations}

As mentioned above, while winds are ubiquitous in AGN, both their effect on AGN host galaxies and their cumulative effect on galaxy evolution is poorly understood. To gain more insight on this topics we collected wind, AGN and host galaxy data for 94 AGN with massive winds detected at differente scales and ionisation states.

We find a strong correlation between mass outflow rate $\dot{M}_{\mathrm{OF}}$ and AGN bolometric luminosity $L_{\text {bol }}$ for both molecular winds $\left(\dot{M}_{\mathrm{OF}} \propto L_{\mathrm{bol}}^{0.76 \pm 0.06}\right)$ and ionised winds $\left(\dot{M}_{\mathrm{OF}} \propto L_{\mathrm{bol}}^{1.29 \pm 0.38}\right)$. Models implying shocks expanding into an isothermal sphere (density $\propto R^{-2}$ ) predict $\dot{M}_{\mathrm{OF}} \propto L_{\text {bol }}^{1 / 3}$ (Faucher-Giguere \& Quataert 2012; King \& Pounds 2015). Lapi et al. (2005) finds a $\dot{M}_{\mathrm{OF}} \propto L_{\mathrm{bol}}^{0.5}$ scaling in the case of an isothermal density profile, if the ratio between the outflow energy and the energy of the ambient ISM $\Delta E / E$ is kept constant. Steeper scalings can be obtained for shocks expanding in a medium where the density profile is flatter than the isothermal case (Faucher-Giguere \& Quataert 2012). However, it should be considered that all quoted models refer to a total mass outflow rate and do not consider multiphase winds, while our determinations concern a particular wind phase (neutral-molecular or atomic-ionised).

Because the scaling of $\dot{M}_{\mathrm{OF}}$ with $L_{\mathrm{bol}}$ of ionised winds is steeper than that of molecular wind, the ratio between molecular and ionised mass outflow rate reduces toward high bolometric luminosity. For the sources of our sample with molecular gas estimates we also find that the molecular gas depletion timescale and the molecular gas fraction, both corrected for the trends with the redshift and with the distance from the main sequence according to the recipe of Genzel et al. (2015), are 3-30 times shorter and $\sim 10$ times smaller, respectively, than the average of star-forming galaxies with similar SFR, stellar mass, distance from the main sequence and redshift. One may speculate then that at high AGN bolometric luminosity and wind kinetic power, the reduced molecular gas fraction may be due to the destruction of molecules in the wind, leading to a large fraction of gas in the atomic/ionised phase. Indeed, models of molecular shocks at densities $>10^{4} \mathrm{~cm}^{-3}$ predicts that the shock will dissociate $\mathrm{H}_{2}$ and become $J$ type (Draine et al. 1983). Models of $J$-type shocks predict efficient reformation of molecules in the post-shock gas, as well as UV radiation to photo-dissociate CO and significant HCN formation (Neufeld \& Dalgarno 1989). Interestingly, 
where observations sensitive enough to detect HCN do exist, (e.g. Markarian 231), enhanced HCN broad wings have been revealed (Aalto et al. 2012, 2015).

We find a strong correlation between $v_{\max }$ and the AGN bolometric luminosity for extended molecular winds (mostly $\mathrm{CO}$ winds), ionised winds and X-ray UFOs. The scaling of $v_{\max }$ with $L_{\mathrm{bol}}$ of molecular+ionised winds is similar to that of UFOs. Both scalings between bolometric luminosity and maximum velocity are consistent with $v_{\max }$ to the fifth power, similar to the MBH- $\sigma$ scaling. It is interesting to note that this scaling is similar to that predicted by Costa et al. (2015) for the case of energy conserving outflows (see their Eqs. (16) and (17)).

It is instructive to compare the latter results with those of samples of similar (or even bigger) size from Herschel spectroscopy of $\mathrm{OH}$ lines. Relative to $\mathrm{CO}$, the specific characteristic of $\mathrm{OH}$ in galaxies is that lines are likely radiatively (instead of collisionally) excited, and thus selectively trace a warmer outflow region, closer to the circum-nuclear source of strong far-IR radiation density. Although the outflow is not spatially resolved the observed excitation conditions provide information about the spatial extent of the outflow, which enables the estimation of the outflow physical parameters (mass-outflow rate, mechanical power and energy).

Gonzalez-Alfonso et al. (2014) present a detailed analysis of $\mathrm{OH}$ signal in Markarian 231. The blue wing of the absorption detected in the high-lying $65 \mu \mathrm{m}$ doublet with $E_{\text {lower }}=290 \mathrm{~K}$, with high-velocity shifts $>1000 \mathrm{~km} \mathrm{~s}^{-1}$, indicates that the excited outflowing gas is generated in a compact and warm (circum)nuclear region (diameter of a few hundred pc). Aalto et al. (2012) and Cicone et al. (2012) found that the molecular outflow size in Markarian 231 decreases with the critical density (it is smaller for higher $\mathrm{CO}$ transition and for $\mathrm{HCN}$ ). $\mathrm{OH}$ transitions may lie on this trend. Furthermore, $\mathrm{OH}$ outflow velocities and mass-outflow rates are similar to that derived from $\mathrm{CO}$ in the few sources where both $\mathrm{CO}$ and $\mathrm{OH}$ winds are detected simultaneously (see the Appendix).

Spoon et al. (2013) analysed the $79 \mu \mathrm{m}$ and $119 \mu \mathrm{m} \mathrm{OH}$ transitions in a sample of 24 ULIRGs at $z<0.26$. Veilleux et al. (2013) analysed the $\mathrm{OH} 119 \mu \mathrm{m}$ transition in 43 mergers at $z<0.3$, mostly ULIRGs and QSOs (six objects are in common with the Spoon et al. 2013, sample). Both works found that outflows are a common (seen in $>70 \%$ of the cases), and that the relative strength of the $\mathrm{OH}$ emission component decreases as the silicate $9.7 \mu \mathrm{m}$ absorption increases, locating the $\mathrm{OH}$ outflows inside the obscured nuclei. Both authors also found that the outflow velocity does not correlate with the galaxy SFR while it correlates with AGN bolometric luminosity, suggesting that, at least in ULIRGs and QSOs, AGN dominates over star formation in driving the outflow (also see Cicone et al. 2014).

More recently, Stone et al. (2016) searched for outflowing $\mathrm{OH}$ in 52 local (distance $<50 \mathrm{Mpc}$ ) AGN, selected at hard X-ray wavelengths by Swift BAT. While $\mathrm{OH}$ is detected in absorption in 17 cases, outflows $\left(v_{84}<-300 \mathrm{~km} \mathrm{~s}^{-1}\right)$ are detected in four cases only (detection rate of $24 \%$ ). Combining this sample with that of Veilleux et al. (2013), Stone et al. confirm the trend of outflow velocity with AGN bolometric luminosity. Furthermore, increasing by several orders of magnitude the dynamic range in SFR, a trend of outflow velocity with SFR emerges, suggesting that at low AGN bolometric luminosities both AGN and star formation contribute in driving $\mathrm{OH}$ outflows (see also Gonzalez-Alfonso et al. 2017).

We report in Fig. 2 the $L_{\text {bol }}-v_{\max }$ (and $L_{\text {bol }}-v_{84}$ ) scalings found by Spoon et al. (2013) and Veilleux et al. (2013) for $\mathrm{OH}$ outflows. While the Veilleux et al. scalings agree quite well with our results, the Spoon et al. (2013) scaling is somewhat flatter than both the Veilleux et al. (2013) scaling and our scalings. We note that the dynamic range in bolometric luminosity covered by Spoon et al. (2013) is smaller than that of Veilleux et al. (2013) and much smaller than ours, and that the uncertainty on the slope of a correlation depends linearly on the dynamic range. Unfortunately, Stone et al. (2016) do not publish a best fit correlation between $v_{84}$ and $L_{\text {bol }}$. However, we mark in Fig. 2 the loci covered by two groups of Swift BAT AGN with $42.3<L_{\text {bol }}<43.3$ and $43.3<L_{\text {bol }}<43.3$ and by the outlier NGC 7479. We note that the Stone et al. results on low luminosity AGN align reasonably well along the correlation found for our sample, once allowing for the offset between $v_{84}$ and $v_{\max }$.

If the scaling of $v_{\max }$ with $L_{\mathrm{bol}}$ of molecular+ionised winds is similar to that of UFOs, than at each given bolometric luminosity the ratio between UFO maximum velocity and molecularionised wind maximum velocity should be similar and equal to $\sim 40-50$. This implies that the gas mass involved in Galaxy scale outflows should be 1500-2500 times the gas mass involved in nuclear high velocity winds. This prediction can be verified by measuring in the same objects both the nuclear and the galaxy scale winds. So far this has been possible in three AGN only, Markarian 231 (Feruglio et al. 2015), IRAS F11119+13257 (Tombesi et al. 2015) and APM08279 (Feruglio et al., in prep.). In these three cases the ratio between the gas mass of the nuclear and galaxy scale winds is in the right ballpark, but more observations of this kind are clearly needed before drawing a strong conclusion (see also Stern et al. 2016).

\subsection{AGN winds in a cosmological context}

We use the continuity equation to compute the evolution of the SMBH accretion rate and compare it to the cosmic SFR density. We find that, the ratio of the average SMBH mean accretion density and average SFR density is about constant with redshift. In massive galaxies the ratio is about constant for constant Eddington ratio distributions and constant radiative efficiencies, while it decreases with increasing redshift for an evolving Eddington ratio distribution and a mass dependent radiative efficiency. For less massive galaxies the ratio peaks at $z \sim 0.5-$ 0.7 in all studied cases.

We evaluate the evolution of the average AGN wind massloading factor, $\langle\eta\rangle$, the relative importance of AGN winds to deprive star formation from its fuel, by convolving the AGN wind - AGN bolometric luminosity scaling relation with AGN bolometric luminosity density, and dividing the result for the SFR density. We find that if $\dot{M}_{\mathrm{OF}} \propto L_{\mathrm{bol}}^{0.76}$, as suggested by molecular winds, $\langle\eta\rangle$ is between 0.2 and 0.3 for the full galaxy population. Instead, $\langle\eta\rangle>1$ for massive galaxies at $z \lesssim 2$. A tentative conclusion is then that AGN winds are, on average, powerful enough to clean galaxies from their molecular gas (either expelling it from the galaxy or by destroying the molecules) in massive systems at least at $z \lessgtr 2$. At higher redshifts the uncertainties in both wind mass outflow rate density and SFRD are today too big to derive solid conclusios. Should the scaling between $\dot{M}_{\mathrm{OF}}$ and $L_{\mathrm{bol}}$ be steeper than assumed above, $\langle\eta\rangle$, would be higher. The steep rise of $\langle\eta\rangle$ between $z=1$ and $z=0.2$ for massive galaxies is due to the equally steep decrease of the SFRD in these systems. We caution that our results are obtained using a crude splitting of galaxies into two broad groups, and, of course, the results are sensitive to the particular galaxy mass threshold adopted for the splitting, in particular where the trends are steeper.

We remark again that all results presented above are based on heterogeneous and biased AGN wind samples. In particular, the 
relationships between molecular wind properties and AGN/host galaxy properties are calibrated at low-redshift only. We assume that similar scalings hold up to $z=2-3$, which is something that only new deep ALMA, NOEMA and VLA observations can confirm. Despite these limitations, our results suggest that AGN wind kinetic energy rate and mass-loading factor can be large in single systems. They may still be important when diluting their effect by accounting for the short AGN phases compared to the star formation cosmic timescales. AGN winds may be the long-sought smoking gun of AGN feedback in action in massive galaxies at $z \lessgtr 2$, while at smaller masses other mechanisms are also likely to be in place (e.g. Peng et al. 2015).

The relationship between AGN winds and SFR does not appear to be simple, even in the best studied systems with the strongest winds. The idea that AGN driven winds may simply clean their host galaxies from dense gas, thus stopping the formation of any new star, is probably an over-simplistic view of a very complex, non-linear process. Winds inject energy and entropy in the ISM, ionising and heating it up. Outflowing gas may experience different phases, as our results suggest (but note that it is not at all clear how dense cold molecular gas can be involved in these winds, see Ferrara \& Scannapieco 2016). A fraction may leave the system and pollute the circum-galactic medium, but some may rain back into the galaxy disk. The gas leaving the galaxy may inject energy, entropy and metals into the circum-galactic medium (CGM), thus affecting the cooling of the CGM gas and, in doing so, affecting further gas accretion into the galaxy. AGN feedback is then likely part of a complex feeding and feedback cycle, consistent with a strong form of AGN/galaxy co-evolution. Gas cools down forming stars and accreting toward the nucleus, giving rise to the growth of the central SMBH through luminous AGN phases. In turn, the AGN powers winds that can heat both ISM and the CGM, altering further star formation and nuclear gas accretion. The SMBH growth is then stopped, as well as nuclear activity and winds, until new cold gas accretes toward the nucleus, so starting a new AGN episode. In this cycle winds and feedbacks might be identified with the "growth hormone" of galaxies, that regulates and modulates galaxy and $\mathrm{BH}$ growth.

Finally, AGN winds may help in cleaning the way through their host galaxy, by both removing the gas, and by ionising it through shocks and high energy radiation, thus allowing ionising photons from both the AGN and the star-forming regions to escape in the IGM (Giallongo et al. 2015). This may contribute to the ionising UV background at high- $z$, which is eventually the responsible for re-ionising the Universe at $z=6-8$.

\section{Conclusions}

We collected multiwavelength observations of 94 AGN host galaxies at various redshifts, characterised by the presence of a wind detected in a given gas phase. We used these observations to study the scaling relationships between wind properties, AGN properties and host galaxy properties. We report the following findings:

1. We confirm, over the largest sample available to date, the remarkable correlation between mass outflow rate and AGN bolometric luminosity (Fig. 1, left panel). For molecular winds $\dot{M}_{\mathrm{OF}} \propto L_{\mathrm{bol}}^{0.76 \pm 0.06}$, while for ionised winds the scaling is $\dot{M}_{\mathrm{OF}} \propto L_{\mathrm{bol}}^{1.29 \pm 0.38}$ (Table 1). These scalings are steeper than those predicted by shock models expanding into a medium with an isothermal density profile. Flatter density profiles may help in explaining the observed scaling.
2. The scaling of $\dot{M}_{\mathrm{OF}}$ with $L_{\mathrm{bol}}$ is steeper for ionised winds than for molecular winds, meaning that the ratio between molecular and ionised mass outflow rates reduces at the highest AGN bolometric luminosities, i.e. the fraction of outflowing gas in the ionised phase increases with the bolometric luminosity.

3. The wind kinetic energy rate $\dot{E}_{\text {kin }}$ is correlated with $L_{\text {bol }}$ (Fig. 1, right panel) for both molecular and ionised outflows $\left(\dot{E}_{\text {kin }} / L_{\text {bol }} \sim 1-10 \%\right.$ for molecular winds, $\dot{E}_{\text {kin }} / L_{\text {bol }} \sim$ $0.1-10 \%$ for ionised winds). About half X-ray absorbers and BAL winds have $\dot{E}_{\text {kin }} / L_{\text {bol }} \sim 0.1-1 \%$ with another half having $\dot{E}_{\text {kin }} / L_{\text {bol }} \sim 1-10 \%$. A few UFOs may have $\dot{E}_{\text {kin }} \sim L_{\text {bol }}$, although the uncertainties in the estimate $\dot{E}_{\text {kin }}$ of UFOs are quite large.

4. $v_{\max }$ correlates with the bolometric luminosity for molecular+ ionised winds and for UFOs (Fig. 2, left panel). Both scalings are statistically consistent with each other, implying that, at each given bolometric luminosity, the ratio between UFO maximum velocity and molecular-ionised wind maximum velocity is $\sim 40-50$ and that the total gas mass involved in Galaxy scale outflows should be 1500-2500 times the gas mass involved in nuclear high velocity winds.

5. The momentum load of most molecular winds is $\dot{P}_{\mathrm{OF}} / \dot{P}_{\mathrm{AGN}}>3$, half have momentum load $>10$, pointing toward molecular winds observed in the energy conserving phase. About half ionised winds have momentum load $<1$ with the other half having $\dot{P}_{\mathrm{OF}} / \dot{P}_{\mathrm{AGN}}>1$ and a few $>10$, suggesting that several ionised winds may also be energy conserving. BAL winds and X-ray absorbers have momentum load in the range $0.01-1$. Fast $X$-ray winds may be identified with the momentum conserving, semi-relativistic wind phase, occurring on scales close to the accretion disc. BAL winds share similar velocities and momentum load of warm absorbers (Fig. 2, right panel).

6. Similar to other studies, we found that most molecular winds and the majority of ionised winds have kinetic power in excess to what would be predicted if they were driven by $\mathrm{SNe}$, based on the SFR measured in the AGN host galaxies (Fig. 3, left panel). The straightforward conclusion is that most powerful winds are AGN driven.

7. The AGN wind mass-loading factor, $\eta=\dot{M}_{\mathrm{OF}} / S F R$, is not strongly correlated with the AGN bolometric luminosity (Fig. 4, left panel) and is systematically higher than the massloading factor of starburst driven winds at each given galaxy stellar mass (Fig. 4, right panel).

8. The depletion timescales and gas fractions of galaxies hosting strong winds are 3-30 times shorter and 10 smaller, respectively, than the average of star-forming galaxies with similar SFR, stellar mass, distance from the main sequence and redshift (Figs. 6 and 7).

We then attempted to put AGN winds into a broader cosmological framework to assess the relative importance of AGN winds on the average SFR, accounting for the short AGN duty cycle. We can summarise the results as follows:

1. We find that the ratio of the average SMBH mean accretion density and average SFR density is about constant with redshift. In massive galaxies, the ratio is about constant for constant Eddington ratio distributions and constant radiative efficiencies, while it decreases with increasing redshift for an evolving Eddington ratio distribution and a mass dependent radiative efficiency. For less massive galaxies the ratio peaks at $z \sim 0.5-0.7$ in all studied cases (Fig. 11). 
2. Finally, we find that the average AGN wind mass-loading factor, $\langle\eta\rangle$ is between 0.2 and 0.3 for the full galaxy population while $\langle\eta\rangle>1$ for massive galaxies at $z \lesssim 2$ (Fig. 12). A tentative conclusion is then that AGN winds are, on average, powerful enough to clean galaxies from their molecular gas (either expelling it from the galaxy or by destroying the molecules) in massive systems only, and at $z \lesssim 2$.

AGN wind studies are evolving from childhood to adult age, and much remains to be understood. The next step is targeting unbiased AGN and galaxy samples, thus deriving direct information on wind demography. This is a difficult and time consuming effort which several on-going programs aim at achieving in the next years (VLT/KMOS KASHz and KMOS3D surveys, VLT/SINFONI SUPER survey, VLT/SINFONI, LBT/LUCI WISSH survey, IRAM PHIBBS2 and IBISCO surveys). In particular, it is crucial to push to high redshift the systematic study of molecular winds. All this will allow us to measure wind parameters and SFR in well defined and little biased samples of AGN at different redshifts, and calculate first the wind massloading factor source by source, and then its average over each redshift range. Then, we need to assess whether the winds are typically multiphase winds, and/or different wind phases are geometrically distinct. Finally we need to understand the fate of the outflowing gas, whether it remains in the systems or if it reaches the CGM.

Acknowledgements. This work was supported by ASI/INAF contract I/009/10/0 and INAF PRIN 2011, 2012 and 2014. M.B. acknowledges support from the FP7 Career Integration Grant "eEASy" (CIG 321913). L.Z. acknowledges support from ASI/INAF grant I/037/12/0. C.F. acknowledges funding from the European Union Horizon 2020 research and innovation programme under the Marie Sklodowska-Curie grant agreement No. 664931. C.C. acknowledges funding from the European Union Horizon 2020 research and innovation programme under the Marie Sklodowska-Curie grant agreement No. 664931 and support from Swiss National Science Foundation Grants PP00P2_138979 and PP00P2_166159. R.M. acknowledges the ERC Advanced Grant 695671 QUENCH and support from the Science and Technology Facilities Council (STFC). F.F. thanks Stefano Borgani and Silvano Molendi for useful discussions. We thank an anonymous referee for comments that helped to improve the presentation.

\section{References}

Aalto, S., Garcia-Burillo, S., Muller, S., et al. 2012, A\&A, 537, A44 Aalto, S., Garcia-Burillo, S., Muller, S., et al. 2015, A\&A, 574, A85 Aird, J., Nandra, K., Laird, E. S., et al. 2010, MNRAS, 401, 2531 Aird, J., Coil, A. L., Georgakakis, A., et al. 2015, MNRAS, 451, 1892 Alatalo, K., Bliz, L., Young, L. M., et al. 2011, ApJ, 735, 88 Alatalo, K., Nyland, K, Greves, G., et al. 2014, ApJ, 780, 186 Alexander, D. M., Vignali, C., Bauer, F. E., et al. 2002, AJ, 123, 1149 Alexander, D. M., Swinbank, A. M., Smail, I., McDermid, R., \& Nesvadba, N. P. H. 2010, MNRAS, 402, 2211

Bahcall, J. N., Kirhakos, S., Saxe, D. H., \& Schneider, D. P. 1997, ApJ, 479, 642 Bautista, M. A., Dunn, J. P., Arav, N., et al. 2010, ApJ, 713, 25 Bischetti, M., Piconcelli, E. Vietri, G., et al. 2017, A\&A, 598, A122 Bolatto, A, Wolffire, M., \& Leroy, A. K. 2013, ARA\&A, 51, 207 Bongiorno, A., Zamorani, G. Gavignaud, I., et al. 2007, A\&A, 472, 443 Bongiorno, A., Merloni, A., Brusa, M., et al. 2012, MNRAS, 427, 310 Bongiorno, A., Schultze, A., Merloni, A., et al. 2016, A\&A, 588, A78 Borguet, C. J., Arav, N., Edmonds, D., Chamberlain, C., \& Benn, C. 2013 ApJ, 762,49

Bouwens, R. J., Illingworth, G. D., Oesch, P. A., et al. 2011, ApJ, 737, 90 Bouwens, R. J., Illingworth, G. D., Oesch, P. A., et al. 2015, ApJ, 803, 34 Brandt, N. W., \& Hasinger, G. 2005, ARA\&A, 43, 827 Brandt, N. W., \& Alexander, D. M. 2015, A\&ARv, 23, 93

Brusa, M., Comastri, A., \& Daddi, E. 2005, A\&A, 432, 69 Brusa, M., Fiore, F., Santini, P., et al. 2009, A\&A, 507, 1277 Brusa, M., Civano, F., Comastri, A., et al. 2010, ApJ, 716, 348 Brusa, M., Bongiorno, A., Cresci, G., et al. 2015a, MNRAS, 446, 2394 Brusa, M., Feruglio, C., Cresci, G., et al. 2015b, A\&A, 578, A11 Brusa, M., Perna, M., Cresci, G., et al. 2016, A\&A, 588, A11
Cano-Diaz, M., Maiolino, R., Marconi, A., et al. 2012, A\&A, 537, A8 Carniani, S., Marconi, A., Maiolino, R., et al. 2015, A\&A, 580, A102 Carniani, S., Marconi, A., Maiolino, R., et al. 2016, A\&A, 591, A28 Cattaneo, A. Faber, S. M., Binney, J., et al. 2009, Nature, 460, 213 Cavagnolo, K. W., Donahue, M., Voit, G. M., \& Sun, M. 2008, ApJ, 683, 107 Cavagnolo, K. W., Donahue, M., Voit, G. M., \& Sun, M. 2009, ApJS, 182, 12 Cavaliere, A., Morrison, P., \& Wood, K. 1971, ApJ, 170, 223

Chartas, G., Saez, C., Brandt, W. N., Giustini, M., \& Garmire, G. P. 2009, ApJ, 706, 644

Cicone, C., Feruglio, C., Maiolino, R., et al. 2012, A\&A, 543, A99

Cicone, C., Maiolino, R., Gallerani, S., et al. 2014, A\&A, 562, A21 Cicone, C., Maiolino, R., Gallerani, S., et al. 2015, A\&A, 574, A14 Cimatti, A., Brusa, M., Talia, M., et al. 2013, ApJ, 779, 13

Combes, F., Garcia-Burillo, S., Casasola, V., et al. 2013, A\&A, 558, A124 Costa, T., Sijacki, D., \& Haehnelt, M. G. 2015, MNRAS, 448, 30

Cowie, L., Songalia, A., Hu, E., \& Cohen, J. G. 1996, AJ, 112, 839 Cresci, G., Mainieri, V., \& Brusa, M. 2015, ApJ, 799, 82 da Cunha, E, Charmandaris, V., Diaz-Santos, T., et al. 2010, A\&A, 523, A78 Daddi, E., Dickinson, M., Morrison, G., et al. 2007, ApJ, 670, 156 Dasyra, K., Tacconi, L. J., Davies, R. I, et al. 2006, ApJ, 651, 835 Davies, R. I., Tacconi, L. J., \& Genzel, R. 2004, ApJ, 613, 781 Davies, R. I., Muller Sanchez, F., Genzel, R., et al. 2007, ApJ, 671, 1388 de Kool, M., Becker, R. H., Gregg, M. D., White, R. L., \& Arav, N. 2002, ApJ, 567,58

Downes, D., \& Solomon, P. M. 1998, ApJ, 507, 615

Draine, B. T., Roberge, W. G., \& Dalgarno, A. 1983, ApJ, 264, 485

Dunlop, J. S., McLure, R. J., Kukula, M. J., et al. 2003, MNRAS, 340, 1095 Dunn, J., Crenshaw, D. M., Kraemer, S. B., \& Trippe, M. L. 2010, ApJ, 713, 900 Duras, F., Bongiorno, A., Piconcelli, E., et al. 2017, A\&A, submitted Dutton, A. A., \& van den Bosch, F. C. 2009, MNRAS, 396, 141 Ebrero, J., Carrera, F. J., Page, M. J., et al. 2009, A\&A, 493, 55 Elvis, M. 2000, ApJ, 545, 63

Engel, H., Davies, R. I., Genzel, R., et al. 2010, A\&A, 524, A56 Fabian, A. C. 1999, MNRAS, 308, 39

Fabian, A. C. 2012, ARA\&A, 50, 455

Faucher-Giguere, C. A., \& Quataert, E. 2012, MNRAS, 425, 605 Ferrara, A., \& Scannapieco, E. 2016, ApJ, 833, 46

Ferrarese, L., \& Ford, H. 2005, Space Sci. Rev., 116, 523

Feruglio, C., Maiolino, R., Piconcelli, E., et al. 2010, A\&A, 518, A155 Feruglio, C., Fiore, F., Maiolino, R., et al. 2013a, A\&A, 549, A51

Feruglio, C., Fiore, F., Piconcelli, E., et al., et al. 2013b, A\&A, 558, A87 Feruglio, C., Fiore, F., Carniani, S., et al. 2015, A\&A, 583, A99 Fiore, F., Brusa, M., Cocchia, F., et al. 2003, A\&A, 409, 79

Fiore, F., Puccetti, S., Grazian, A., et al. 2012, A\&A, 537, A16 Fischer, J., Sturm, E., Gonzales-Alfonso, E., et al. 2010, A\&A, 518, A41 Franceschini, A., Hasinger, G., Miyaji, T., \& Malguori, D. 1999, MNRAS, 310, L5

Garcia-Burillo, S., Combes, F., Usero, A., et al. 2014, A\&A, 576, A125 Gaspari, M., \& Sadowski, A. 2017, ApJ, 837, 149

Gaspari, M., Brighenti, F., \& Temi, P. 2012, MNRAS, 424, 190 Gaspari, M., Ruszkowski, M., \& Oh, S. P. 2013, MNRAS, 432, 340

Gaspari, M., Brighenti, F., \& Temi, P. 2014, ApJ, 783, 10

Gebhardt, K., Bender, R., Bower, G., et al. 2000, ApJ, 539, L13

Georgakakis, A., Perez-Gonzalez, P. G., Fanidakis, N., et al. 2014, MNRAS, 440, 339

Georgakakis, A., Aird, J., Buchner, J., et al. 2015, MNRAS, 453, 1946 Genzel, R., Tacconi, L. J., Garcia-Caprio, J., et al. 2010, MNRAS, 407, 2091 Genzel, R., Forster-Schreiber, N. M., Rosario, D., et al. 2014, ApJ, 796, 7 Genzel, R., Tacconi, L. J., Lutz, D., et al. 2015, ApJ, 800, 20

Giallongo, E., Grazian, A., Fiore, F., et al. 2015, A\&A, 578, A83

Gonzalez-Alfonso, E., Fisher, J, Gracia-Carpio, J., et al. 2014, A\&A, 561, A27 Gonzalez-Alfonso, E., Fischer, J., Spoon, H. W. W., et al. 2017, ApJ, 836, 11

Granato, G. L., De Zotti, G., Silva, L., Bressan, A., \& Danese, L. 2004, ApJ, 600,580

Greene, J. E., Zakamska, N. L., \& Smith, P. S. 2012, ApJ, 746, 86

Gruppioni, C., Calura, F., Pozzi, F., et al. 2015, MNRAS, 451, 3419

Hopkins, P. F., Hernquist, L., Cox, T. J., et al. 2006, ApJS, 163, 1

Harrison, C. M., Alexander, D. M., Swinbank, A. M., et al. 2012, MNRAS, 426, 1073

Harrison, C. M., Alexander, D. M., Mullaney, J. R., \& Swinbank, A. M. 2014, MNRAS, 441, 3306

Heckman, T. M., Alexandroff, R. M., Borthaukur, S., Overzier, R., \& Leitherer, C. 2015, ApJ, 809, 147

Hickox, R. C., Mullaney, J. R., Alexander, D. M., et al. 2014, ApJ, 782, 9

Howell, J. H., Armus, L., Mazzarella, J. M., et al. 2010, ApJ, 715, 572

Jahnke, K., \& Maccio, A. V. 2011, ApJ, 734, 92

Jahnke, K., Sanchez, S. F., Wisotski, L., et al. 2004, ApJ, 614, 568

Jin, C., Ward, M., \& Done, C. 2012, MNRAS, 425, 907 
Kaastra, J., Kriss, J. A., Cappi, M., et al. 2014, Science, 345, 64

Kalfountzou, E., Civano, F., Elvis, M., Trichas, M., \& Green, P. 2014, MNRAS 445,1430

King, A. R. 2003, ApJ, 596, 27

King, A. R., \& Pounds, K. 2015, ARA\&A, 53, 115

Korista, K. T., Bautista, M. A., Arav, N., et al. 2008, ApJ, 688, 108

Kormendy, J., \& Ho, L. C. 2013, ARA\&A, 51, 511

Krips, M., Martin, S., Eckart, A., et al. 2011, ApJ, 736, 37

La Franca, F., Fiore, F., Comastri, A., et al. 2005, ApJ, 635, 864

Lanzuisi, G., Giustini, M., Cappi, M., et al. 2012, A\&A, 544, A2

Lapi, A., Cavaliere, A., \& Menci, N. 2005, ApJ, 619, 60

Lipari, S., Sanchez, S. F., Bergmann, M., et al. 2009, MNRAS, 392, 1295

Lonsdale, C. J., Smith, H. E., \& Diamond, P. J. 2003, ApJ, 592, 804

Liu, G., Zakamska, N. L., Greene, J. E., Nesvadba, N. P. H., \& Liu, X. 2013a, MNRAS, 430, 2327

Liu, G., Zakamska, N. L., Greene, J. E., Nesvadba, N. P. H., \& Liu, X. 2013b, MNRAS, 436, 2576

Madau, P., \& Dickinson, M. 2014, ARA\&A, 52, 415

Mainieri, V., Bongiorno, A., Merloni, A., et al. 2011, A\&A, 535, A80

Marconi A., Risaliti, G., Gilli, R., et al. 2004, MNRAS 351, 169

Maiolino, R., Gallerani, S., Neri, R., et al. 2012, MNRAS, 425, 66

Malizia, A., Landi, R., Bassani, L., et al. 2007, ApJ, 668, 81

Mancuso, C., Lapi, A., Shi, J., et al. 2016, ApJ, 833, 152

Marchesi, S., Civano, F., Elvis, M., et al. 2016, ApJ, 817, 34

McNamara, B. R., \& Nulsen, P. E. J. 2007, ARA\&A, 45, 117

Menci, N., Cavaliere, A., Fontana, A., et al. 2003, ApJ, 587, 63

Merloni, A., \& Heinz, S. 2008, MNRAS, 388, 1011

Merloni, A., Rudnick, G., \& Di Matteo, T. 2004, MNRAS, 354, L37

Mignoli, M., Pozzetti, L., Comastri, A., et al. 2004, A\&A, 418, 827

Moe, M., Arav, N., Bautista, M., \& Korista, K. T. 2009, ApJ, 706, 525

Morganti, R., Oosterloo, T., \& Tsvetanov, Z. 1998, AJ, 115, 915

Morganti, R., Fogasy, J., Paragi, Z., Oosterloo, T., \& Orienti, M. 2013a, Science, 341,1082

Morganti, R., Frieswijk, W., OOnk, R. J. B., Oosterloo, T., \& Tadhunter, C. 2013b, A\&A, 552, A4

Mullaney, J. R., Daddi, E., Béthermin, M., et al. 2012, ApJ, 753, L30

Mullaney, J. R., Alexander, D. M., Aird, J., et al. 2015, MNRAS, 453, 83

Nandra, K., Georgakakis, A., Willmer, C. N. A., et al. 2007, ApJ, 660, 11

Nandra, K., Barret, D., Barcons, X., et al. 2013, ArXiv e-prints [arXiv: 1306.2307]

Nardini, E., Reeves, J., Gofford, J., et al. 2015, Science, 347, 860

Nesvadba, N. P. H., Lehnert, M. D., Eisenhauer, F., et al. 2006, ApJ, 650, 693

Nesvadba, N. P. H., Lehnert, M. D., De Breuck, C., Gilbert, A. M., \& van Breugel, W. 2008, A\&A, 491, 407

Netzer, H. 2009, MNRAS, 399, 1907

Neufeld, D. A., \& Dalgarno, A. 1989, ApJ, 340, 869

Osterbrock D. E., \& Ferland G. J. 2006, Astrophysics of gaseous nebulae and active galactic nuclei (Sausalito, CA: University Science Books)

Peng, C. Y. 2007, ApJ, 668, 513

Peng, Y., Lilly, S., Kovac, K., et al. 2010, ApJ, 721, 193

Peng, Y., Maiolino, R., \& Cochrane, R. 2015, Nature, 521, 192

Perna, M., Brusa, M., Cresci, G., et al. 2015a, A\&A, 574, A82

Perna, M., Brusa, M., Salvato, M., et al. 2015b, A\&A, 583, A72

Piconcelli, E., Jimenez-Bailon, E., Guainazzi, M., et al. 2005, A\&A, 432, 15

Reyes, R., Zakamska, N. L., \& Strauss, M. A., 2008, AJ, 136, 2373
Richstone, D., Ajhar, E. A., Bender, R., et al. 1998, Nature, 395, 14 Riffel, R. A., \& Storchi-Bergmann, T. 2011, MNRAS, 417, 2752 Rodighiero, G., Daddi, E., Baronchelli, I., et al. 2011, ApJ, 739, 40 Rodighiero, G., Brusa, M., Daddi, E., et al. 2015, ApJ, 800, 10

Rosario, D. J., Santini, P., Lutz, D., et al. 2012, A\&A, 545, A45

Rupke, D., \& Veilleux, S. 2011, ApJ, 729, 27

Rupke, D., \& Veilleux, S. 2013, ApJ, 768, 75

Santini, P., Fontana, A., Grazian, A., et al. 2009, A\&A, 504, 75

Sargent, M. T., Daddi, E., Bethermin, M., et al. 2014, ApJ, 793, 19

Shankar, F., Salucci, P., Granato, G. L., De Zotti, G., \& Danese, L. 2004 MNRAS, 354, 1020

Shankar, F., Weinberg, D. H., \& Miralda-Escudé, J. 2009, ApJ, 690, 20

Shankar, F., Weinberg, D. H., \& Miralda-Escudé, J. 2013, MNRAS, 428, 421

Shankar, F., Bernardi, M., Sheth, R. K., et al. 2016, MNRAS, 460, 311

Shankar, F., Bernardi, M., Sheth, R. K. 2017, MNRAS, 466, 4029

Shao, L., Lutz, D., Nordon, R., et al. 2010, A\&A, 518, A26

Shen, Y., Richards, G. T., Strauss, M. A., et al. 2011, ApJS, 194, 45

Schneider, R., Bianchi, S., Valiante, R., Risaliti, G., \& Salvadori, S. 2015, A\&A, 579, A60

Silk, J., \& Rees, M. J. 1998, A\&A, 331, 1

Silverman, J. D., Mainieri, V., Lehmer, B. D., et al. 2008, ApJ, 675, 1025

Small, T. A., \& Blandford, R. D. 1992, MNRAS, 259, 725

Soltan, A. 1982, MNRAS, 200, 115

Somerville, R. S., \& Primack, J. R. 1999, MNRAS, 310, 1087

Spoon, H. W. W., Farrah, D., Lebouteiller, V., et al. 2013, ApJ, 775, 127

Stern, J., Faucher-Giguère, C. A., Zakamska, N., \& Hennawi, J. F. 2016, ApJ, 819,130

Stone, M., Veilleux, S., \& Melendez, M. 2016, ApJ, 826, 111

Sturm, E., Gonzalez-Alfonso, E., Veilleux, S., et al. 2011, A\&A, 518, A36

Sun, A.-L., Greene, J. E., Zakamska, N. L., \& Nesvadba, N. P. H. 2014, ApJ, 190, A160

Tacconi, L. J., Genzel, R., Tecza, M., et al. 1999, ApJ, 524, 732

Tadhunter, C., Morganti, R., Rose, M., Oonk, J. B. R., \& Oosterloo, T. 2014, Nature, 511, 440

Tombesi, F., Sambruna, R. M., Reeves, J. N., et al. 2010, ApJ, 719, 700

Tombesi, F., Cappi, M., Reeves, J., et al. 2013, MNRAS, 430, 1102

Tombesi, F., Melendez, M., Veilleux, S., et al. 2015, Nature, 519, 436

Ueda, Y., Akiyama, M., Otha, K., \& Miyaji, T. 2003, ApJ, 598, 886

Ueda, Y., Akiyama, M., Hasinger, G., Miyaji, T., \& Watson, M. 2014, ApJ, 786, 104

Vasudevan, R. V., \& Fabian, A. C. 2007, MNRAS, 381, 1235

Veilleux, S., Cecil, G., \& Bland-Hawthorn, J. 2005, ARA\&A, 43, 769

Veilleux, S., Rupke, D. S. N., Kim, D.-C., et al. 2009, ApJS, 182, 628

Veilleux, S., Melendez, M., Sturm, E., et al. 2013, ApJ, 776, 27

Vito, F., Gilli, R., Vignali, C., et al. 2014, MNRAS, 445, 3557

Voit, G. M., \& Donahue, M. 2015, ApJ, 799, 1

Whitaker, K. E., van Dokkum, P. G., Brammer, G., \& Franx, M. 2012, ApJ, 754, 29

Woo, J.-H., \& Urry, M. 2002, ApJ, 579, 530

Wylezalek, D., Zakamska, N. L., Liu, G., \& Obied, G. 2016, MNRAS, 457, 745

Xia, X. Y., Gao, Y., Hao, C.-N., et al. 2012, ApJ, 750, 92

Zheng, X. Z., Bell, E. F., Somerville, R. S., et al. 2009, ApJ, 707, 1566

Zakamska, N. L., Hamann, F., Paris, I., et al. 2016, MNRAS, 459, 3144 


\section{Appendix A: Source samples}

The source samples used in this paper are detailed below and presented in Table B.1. As a general rule, we used only AGN for which there is an estimate (or a robust limit) on the physical size of the high velocity gas involved in the wind. This is in fact crucial to obtain an estimate of the outflow rates of mass and kinetic energy.

\section{A.1. Molecular winds}

The bulk of sample of AGN molecular winds is from the compilation of Cicone et al. (2014), and includes AGN in ULIRGs and nearby Seyfert galaxies. We used additional data from Feruglio et al. (2013a,b: NGC 6240), Feruglio et al. (2015: Markarian 231), Krips et al. (2011) and Garcia-Burillo et al. (2014; NGC 1068), Morganti et al. (2013a,b, IC 5066), Alatalo et al. (2011, NGC 1266), Combes et al. (2013; NGC 1433), and Sun et al. (2014; SDSSJ135646.10+102609.0). In all these cases the winds are traced from high velocity wings observed in $\mathrm{CO}(1-0), \mathrm{CO}(2-1), \mathrm{CO}(3-2)$. We also added to this sample winds traced by high velocity $\mathrm{OH}$ from Sturm et al. (2011; I131205453, I14378-3651 and I17208-0014), and Tombesi et al. (2015; IRAS F11119+13257). Two sources have mass outflow rates computed using both $\mathrm{CO}$ and $\mathrm{OH}$ transitions (Markarian 231, Sturm et al. 2011; Gonzalez-Alfonso et al. 2014; Feruglio et al. 2015; and IRAS 08572+3915, Sturm et al. 2011; Cicone et al. 2014). In these two cases the $\mathrm{CO}$ and $\mathrm{OH}$ mass outflow rates are within $20 \%$. Several of the sources with detected high velocity $\mathrm{CO}$ have also detected high velocity $\mathrm{OH}$ in absorption $\left(\mathrm{NGC} 6240, v_{84}(\mathrm{OH})=544 \mathrm{~km} \mathrm{~s}^{-1}, v_{\max }(\mathrm{OH})=1200 \mathrm{~km} \mathrm{~s}^{-1}\right.$, Veilleux et al. 2013; $v_{\max }(\mathrm{CO})=500 \mathrm{~km} \mathrm{~s}^{-1}$, Feruglio et al. $2013 \mathrm{~b} ; \mathrm{I} 10565, v_{84}(\mathrm{OH})=489 v_{\max }(\mathrm{OH})=950$, Veilleux et al. 2013, $v_{\max }(\mathrm{CO})=600 \mathrm{~km} \mathrm{~s}^{-1}$ Cicone et al. 2014; I23365, $v_{84}(\mathrm{OH})=604 \mathrm{~km} \mathrm{~s}^{-1}, v_{\max }(\mathrm{OH})=1300 \mathrm{~km} \mathrm{~s}^{-1}$, Veilleux et al. 2013; $v_{\max }(\mathrm{CO})=600 \mathrm{~km} \mathrm{~s}^{-1}$, Cicone et al. 2014; IC 5063, $v_{84}(\mathrm{OH})=309 \mathrm{~km} \mathrm{~s}^{-1}$, Stone et al. 2016; $v_{\max }(\mathrm{CO})=$ $400 \mathrm{~km} \mathrm{~s}^{-1}$, Morganti et al. 2013). One source with a CO outflow, NGC 1068, does not have strong OH absorption (Stone et al. 2016). It should however be noted that Herschel samples a much bigger region than that on which the $\mathrm{CO}$ outflow has been detected. In conclusion, mass outflow rates and velocities of $\mathrm{CO}$ outflows and $\mathrm{OH}$ outflows seem comparable, although $\mathrm{OH}$ probably traces more compact wind regions than $\mathrm{CO}(1-0)$ (e.g. Spoon et al. 2013; Gonzalez-Alfonso 2014). Indeed, Aalto et al. (2012) and Cicone et al. (2012) found that the molecular outflow size in Markarian 231 decreases with the critical density (it is smaller for higher $\mathrm{CO}$ transition and for $\mathrm{HCN}$ ). $\mathrm{OH}$ transitions may lie on this trend.

\section{A.2. Ionised winds}

Ionised winds are from the sample of Harrison et al. (2014, type 2 AGN at $z<0.2$ ), Rupke \& Veilleux (2013, local ULIRGs), Liu et al. (2013), Cresci et al. (2015), Brusa et al. (2016), Perna et al. (2015a,b; all X-ray selected AGN at $z \sim 1.5$ ), Harrison et al. (2012; ULIRGs at $z=2-3.3$ ), Nesvadba et al. (2008; Radio galaxies at $z=2.2-2.6$ ), Genzel et al. (2014; AGN in star-forming galaxies at $z=2.1-2.4$ ), Carniani et al. (2015; luminous type 1 QSOs at $z=2.5$ ), Bischetti et al. (2017, hyperluminous QSOs at $z=2.5-3.5$ ). In all these cases the wind is traced by high velocity [OIII] $\lambda 5007, \mathrm{H}_{\beta}$ and/or $\mathrm{H}_{\alpha}$. We finally included in the sample the [CII] wind detected in the $z=6.4$
QSO SDSSJ1148 by Maiolino et al. (2012) and Cicone et al. (2015).

\section{A.3. BAL winds}

We used BAL data only for sources where there is an estimate in the literature of the size of the ionised gas cloud responsible for the absorption. In particular, we used QSOs from Borguet et al. (2013), Moe et al. (2009), Dunn et al. (2010), Korista et al. (2008), Shen et al. (2011).

\section{A.4. X-ray winds}

Most X-ray winds are from the compilation of Tombesi et al. (2013), which include fast UFOs and slower warm absorbers. We added to this list Markarian 231 (Feruglio et al. 2015), IRAS 11119 (Tombesi et al. 2015), PDS456 (Nardini et al. 2015) and APM08279 (Chartas et al. 2009).

\section{Appendix B: Estimates of physical quantities}

\section{B.1. Outflows quantities}

Different recipes are used by different authors to calculate physical quantities from observed ones. To make the comparison between different sources as homogeneous as possible, we recomputed the wind mass outflow rates and kinetic power rates given in Table B. 1 by using standard recipes.

The wind mass outflow rate is then computed using the continuity fluid equation:

$\dot{M}_{\mathrm{OF}}=\Omega R_{\mathrm{OF}}^{2} \rho_{\mathrm{OF}} v_{\max }$

where $\rho_{\mathrm{OF}}$ is the average mass density of the outflow, $\mathrm{v}_{\max }$ is the wind maximum velocity and $R_{\mathrm{OF}}$ is the radius at which the outflow rate is computed, and $\Omega$ is the solid angle subtended by the outflow. Assuming a spherical sector, $\rho_{\mathrm{OF}}=3 M_{\mathrm{OF}} / \Omega R_{\mathrm{OF}}^{3}$, then:

$\dot{M}_{\mathrm{OF}}=3 \times v_{\mathrm{max}} \times M_{\mathrm{OF}} / R_{\mathrm{OF}}$.

Accordingly, $\dot{M}_{\mathrm{OF}}$ represents the instantaneous outflow rate of the material at the edge $R_{\mathrm{OF}}$ (i.e. it is a local estimate) and it is three times larger than the total outflow mass divided by the time required to push this mass through a spherical surface of radius $R_{\mathrm{OF}}$. This estimator does not depend on the solid angle $\Omega$ subtended by the outflow. Three key observables then appear in the definition of the mass outflow rate: $v_{\max }, R_{\mathrm{OF}}$ and $M_{\mathrm{OF}}$.

Following Rupke \& Veilleux (2013) we define the maximum wind velocity as the shift between the velocity peak of broad emission lines and the systemic velocity plus 2 times the $\sigma$ of the broad Gaussian component $\left(v_{\max }=\right.$ velocity shift broad $\left.+2 \sigma_{\text {broad }}\right)$. We assigned to each source $\left(v_{\max }\right)$ either using the published value if it exists, or evaluating it from the published spectra (as in the case of NGC 1068, Krips et al. 2011). Estimating the bulk wind velocity from the observed velocities is not straightforward, because the conversion depends by the wind geometry and spatial distribution of velocities, see the discussion in Liu et al. (2013b). In our analysis we assumed that the bulk wind velocity is $\sim$ maximum wind velocity. Other authors suggest that a better proxy for the bulk wind velocity is W80/1.3 (Liu et al. 2013; Harrison et al. 2014), where W80 is the velocity width of the emission lines at the $80 \%$ of the line flux. Several sources of our sample have published W80 and $v_{\max }$ velocities. For these 
Table B.1. AGN wind sample.

\begin{tabular}{|c|c|c|c|c|c|c|c|c|c|c|}
\hline Name $^{a}$ & Redshift & $\begin{array}{l}\log L_{\mathrm{bol}} \\
\mathrm{erg} \mathrm{s}^{-1}\end{array}$ & $\begin{array}{c}\log \dot{M}_{\mathrm{OF}} \\
M_{\odot} / \mathrm{yr}\end{array}$ & $\begin{array}{c}\log \dot{E}_{\text {kin }} \\
\operatorname{erg~s}^{-1}\end{array}$ & $\begin{array}{c}v_{\max } \\
\mathrm{km} \mathrm{s}^{-1}\end{array}$ & $\begin{array}{l}R_{\mathrm{OF}} \\
\mathrm{kpc}\end{array}$ & $\begin{array}{c}\log S F R \\
M_{\odot} / \mathrm{yr}\end{array}$ & $\begin{array}{c}\log M_{*} \\
M_{\odot}\end{array}$ & $\begin{array}{c}\log M_{\mathrm{gas}} \\
M_{\odot}\end{array}$ & Ref. \\
\hline \multicolumn{11}{|c|}{ Molecular (CO) winds } \\
\hline Mrk 231 & 0.04217 & 45.7 & 3 & 44.25 & 750 & 0.3 & 1.00 & 9.11 & 8.88 & $1,2,3,4,5$ \\
\hline Mrk 231 & 0.04217 & 45.7 & 2.84 & 44.21 & 850 & 1 & 2.06 & 9.80 & 9.3 & $1,2,3,4,5$ \\
\hline NGC 6240 & 0.0248 & 44.8 & 2.70 & 43.6 & 500 & 0.6 & 1.23 & 10.11 & 9.3 & $6,7,8,9$ \\
\hline NGC 6240 & 0.0248 & 44.8 & 2.08 & 42.79 & 400 & 5 & 2.18 & 11.59 & 9.83 & $6,7,8,9$ \\
\hline I08572 & 0.05835 & 45.66 & 3.08 & 44.74 & 1200 & 1 & 1.62 & 11.8 & 9.11 & 10,9 \\
\hline I10565 & 0.04311 & 44.81 & 2.48 & 43.54 & 600 & 1.1 & 1.98 & 11.17 & 9.26 & $10,9,11,12$ \\
\hline I23060 & 0.173 & 46.06 & 3.04 & 44.63 & 1100 & 4 & 1.87 & & 10.39 & 10 \\
\hline I23365 & 0.06448 & 44.67 & 2.23 & 43.29 & 600 & 1.2 & 2.14 & 11.15 & 9.47 & $10,9,11,12$ \\
\hline SDSSJ1356 & 0.1238 & 45.1 & 2.54 & 43.44 & 500 & 0.3 & 0.114 & & 8.48 & 13 \\
\hline NGC 1068 & 0.003793 & 43.94 & 2.08 & 42.18 & 200 & 0.1 & 0.204 & 9.30 & 7.8 & $14,15,5$ \\
\hline NGC 1433 & 0.003589 & 43.11 & 1.03 & 40.89 & 150 & 0.05 & -0.538 & 9.48 & 7.7 & 16 \\
\hline IC 5063 & 0.011 & 44 & 1.34 & 42.05 & 400 & 0.5 & -0.260 & & 7.7 & $17,18,19,20$ \\
\hline NGC 1266 & 0.007318 & 43.3 & 1.11 & 41.73 & 360 & 0.45 & 0.204 & 9.59 & 8.6 & 21,22 \\
\hline \multicolumn{11}{|c|}{ Molecular $(\mathrm{OH})$ winds } \\
\hline I13120 & 0.03076 & 44.84 & 2.11 & 43.48 & 860 & 0.2 & 2.22 & 10.49 & 9.76 & 23,24 \\
\hline I14378 & 0.06764 & 45.43 & 2.87 & 44.51 & 1170 & 0.1 & 1.90 & & 9.62 & 23,11 \\
\hline I17208 & 0.04281 & 45.11 & 1.95 & 42.59 & 370 & 0.1 & 2.44 & 11.13 & 9.38 & $25,26,12$ \\
\hline I11119 & 0.189 & 45.18 & 2.90 & 44.41 & 1000 & 0.3 & 2.20 & & 9.95 & 25,26 \\
\hline \multicolumn{11}{|c|}{ Ionised ([OIII $], \mathrm{H}_{\beta}, \mathrm{H}_{\alpha}$, [CII]) winds } \\
\hline SDSSJ0945 & 0.1283 & 45.51 & 1.62 & 43.49 & 1511 & 2.7 & 1.91 & & & 27 \\
\hline SDSSJ0958 & 0.1092 & 45 & 1.1 & 42.47 & 866 & 2.6 & 1.56 & & & 27 \\
\hline SDSSJ1000 & 0.148 & 45.7 & 1.16 & 42.43 & 761 & 4.3 & 1.46 & & & 27 \\
\hline SDSSJ10101 & 0.1992 & 46 & 1.82 & 43.69 & 1523 & 3.9 & 2.08 & & & 27 \\
\hline SDSSJ 10100 & 0.0984 & 45.6 & 1.46 & 43.16 & 1267 & 1.6 & 1.36 & & & 27 \\
\hline SDSSJ1100 & 0.1005 & 46 & 1.65 & 43.3 & 1192 & 1.9 & & & & 27 \\
\hline SDSSJ 1125 & 0.1669 & 45.2 & 0.74 & 42.63 & 1547 & 2.9 & & & & 27 \\
\hline SDSSJ1130 & 0.1353 & 45.11 & 0.3 & 41.38 & 616 & 2.8 & 1.26 & & & 27 \\
\hline SDSSJ1316 & 0.1505 & 45.4 & 1.48 & 43.15 & 1216 & 3.1 & & & & 27 \\
\hline SDSSJ1339 & 0.139 & 44.3 & 0.22 & 41.13 & 505 & 2.5 & & & & 27 \\
\hline SDSSJ1355 & 0.1519 & 45.7 & 0.57 & 41.87 & 797 & 3.5 & & & & 27 \\
\hline SDSSJ1356 & 0.1238 & 45.1 & 1.60 & 43.14 & 1049 & 3.1 & 1.80 & 11.0 & & 27 \\
\hline SDSSJ1430 & 0.0855 & 45.3 & 1.70 & 43.2 & 999 & 1.8 & 0.85 & & & 27 \\
\hline Q1623 & 2.43 & & 2.45 & 44.18 & 1300 & 1.3 & 1.48 & 10.81 & & 28 \\
\hline U3-25105 & 2.29 & & 1.50 & 41.66 & 214 & 1.3 & 1.51 & 10.85 & & 28 \\
\hline GS3-19791 & 2.22 & 45.6 & 3.23 & 44.18 & 530 & 1.3 & 2.17 & 11.31 & & 28 \\
\hline D3a-15504 & 2.38 & & 1.72 & 42.58 & 475 & 1.3 & 1.38 & 11.04 & & 28 \\
\hline GS3-28008 & 2.29 & 45.9 & 2.34 & 42.8 & 300 & 1.3 & 2.03 & 11.36 & & 28 \\
\hline COS43206 & 2.1 & & 2.06 & 42.52 & 300 & 1.3 & 1.64 & 11.4 & & 28 \\
\hline COS11363 & 2.1 & 46.22 & 2.83 & 44.52 & 1240 & 1.3 & 1.62 & 11.28 & & 28 \\
\hline SDSSJ1326 & 3.304 & 47.59 & 3.81 & 45.98 & 2160 & 7 & 2.26 & & & 29,30 \\
\hline
\end{tabular}

Notes. ${ }^{(a)}$ Short name. See quoted bibliography for full names.

References. 1 = Feruglio et al. (2015); 2 = Lonsdale et al. (2003); 3 = Davies et al. (2004), $4=$ Veilleux et al. (2009), $5=$ Davies et al. (2007); $6=$ Feruglio et al. (2013a); $7=$ Tacconi et al. (1999); $8=$ Engel et al. (2010); $9=$ Howell et al. (2010); $10=$ Cicone et al. $(2014) ; 11=$ Dasyra et al. (2006); $12=$ Downes \& Solomon (1998); $13=$ Sun et al. (2014); $14=$ Garcia-Burillo et al. (2014); $15=$ Krips et al. $(2012) ; 16=$ Combes et al. (2013); $17=$ Morganti et al. (1998); $18=$ Morganti et al. (2013); $19=$ Woo \& Urry (2002); $20=$ Malizia et al. (2007); $21=$ Alatalo et al. (2011); $22=$ Alatalo et al. (2014); $23=$ Sturm et al. (2011); $24=$ da Cunha et al. (2010); $25=$ Veilleux et al. (2013); $26=$ Xia et al. (2012); $27=$ Harrison et al. (2014); $28=$ Genzel et al. (2014), assuming $\mathrm{H}_{\alpha} / \mathrm{H}_{\beta}=2.9$, extinction corrected; $29=$ Bischetti et al. (2017); 30=Duras et al. (2017); $31=$ Carniani et al. (2015); $32=$ Harrison et al. (2012); $33=$ Cresci et al. (2015), extinction corrected; $34=$ Brusa et al. $(2015 b) 35=$ Brusa et al. (2015a); $36=$ Perna et al. (2015a), extinction corrected; $37=$ Brusa et al. (2016); $38=$ Perna et al. (2015b); $39=$ Nesvadba et al. (2006), assuming $\mathrm{H}_{\alpha} / \mathrm{H}_{\beta}=2.9$, extinction corrected; $40=$ Nesvadba et al. (2008); $41=$ Rupke \& Veilleux (2013); $42=$ Liu et al. (2013a); $43=$ Liu et al. (2013b), extinction corrected; $44=$ Wylezalek et al. (2016); $45=$ Reyes et al. (2008); $46=$ Maiolino et al. (2012); $47=$ Cicone et al. (2015) [CII $]$ wind; $48=$ Borguet et al. (2013); $49=$ Bandara et al. 2009; $50=$ Moe et al. (2009); $51=$ Dunn et al. (2010); $52=$ Korista et al. (2008); 53=Bautista et al. (2010); $54=$ de Kool et al. (2002); $55=$ Shen et al. (2011); 56 = Tombesi et al. (2012); 57 = Nardini et al. (2015); $58=$ Tombesi et al. (2015); $59=$ Chartas et al. (2009). 
Table B.1. continued.

\begin{tabular}{|c|c|c|c|c|c|c|c|c|c|c|}
\hline Name $^{a}$ & Redshift & $\begin{array}{l}\log L_{\mathrm{bol}} \\
\mathrm{erg} \mathrm{s}^{-1}\end{array}$ & $\begin{array}{c}\log \dot{M}_{\mathrm{OF}} \\
M_{\odot} / \mathrm{yr}\end{array}$ & $\begin{array}{l}\log \dot{E}_{\mathrm{kin}} \\
\operatorname{erg~s}^{-1}\end{array}$ & $\begin{array}{c}v_{\max } \\
\mathrm{km} \mathrm{s}^{-1}\end{array}$ & $\begin{array}{l}R_{\mathrm{OF}} \\
\mathrm{kpc}\end{array}$ & $\begin{array}{c}\log S F R \\
M_{\odot} / \mathrm{yr}\end{array}$ & $\begin{array}{c}\log M_{*} \\
M_{\odot}\end{array}$ & $\begin{array}{c}\log M_{\text {gas }} \\
M_{\odot}\end{array}$ & REF \\
\hline SDSSJ1549 & 2.367 & 47.82 & 3.42 & 45.2 & 1380 & 7 & & & & 29,30 \\
\hline SDSSJ1201 & 3.512 & 47.76 & 3.50 & 45.53 & 1850 & 7 & & & & 29,30 \\
\hline SDSSJ0745 & 3.22 & 47.99 & 3.76 & 45.81 & 1890 & 7 & 3.18 & & & 29,30 \\
\hline SDSSJ0900 & 3.297 & 47.91 & 3.52 & 45.77 & 2380 & 7 & 2.90 & & & 29,30 \\
\hline LBQS0109 & 2.35 & 47.43 & 2.84 & 44.88 & 1850 & 0.4 & & & & 31 \\
\hline 2QZJ0028 & 2.401 & 47.15 & 3.66 & 45.89 & 2300 & 0.7 & 2.00 & & & 31 \\
\hline HB8905 & 2.48 & 46.77 & 2.65 & 43.55 & 500 & 1.3 & & & & 31 \\
\hline HE0109 & 2.407 & 47.39 & 3.14 & 44.55 & 900 & 0.4 & 1.70 & & & 31 \\
\hline HB8903 & 2.44 & 47.28 & 1.76 & 43.58 & 1450 & 1.9 & 1.95 & & & 31 \\
\hline RGJ0302 & 2.239 & 46.34 & 1.48 & 43.17 & 1234 & 8 & 2.93 & & & 32 \\
\hline SMMJ0943 & 3.351 & 46.76 & 1.57 & 43.17 & 1124 & 15 & 3.11 & & & 32 \\
\hline SMMJ1237 & 2.06 & 46.72 & 1.48 & 43.14 & 1200 & 7 & 2.63 & & & 32 \\
\hline SMMJ1636 & 2.385 & 46.28 & 1.44 & 42.99 & 1054 & 7 & 3.15 & & & 32 \\
\hline XID2028 & 1.593 & 46.3 & 2.39 & 44.24 & 1500 & 13 & 2.44 & 11.65 & 10.28 & 33,34 \\
\hline XID5321 & 1.47 & 46.3 & 1.84 & 43.93 & 1950 & 11 & 2.36 & 11.7 & & 35,36 \\
\hline XID5395 & 1.472 & 45.93 & 2.65 & 44.56 & 1600 & 4.3 & 2.57 & 10.89 & & 37 \\
\hline MIRO20581 & 2.45 & 46.6 & 2.29 & 44.55 & 1900 & 4.8 & $<2.5$ & 11.28 & & 38 \\
\hline MRC1138 & 2.2 & 46.6 & 2.39 & 43.7 & 800 & 20 & & 10 & & 39 \\
\hline MRC0406 & 2.44 & 46.3 & 3.82 & 45.29 & 960 & 9.3 & & 8.60 & & 40 \\
\hline MRC0828 & 2.57 & 46.6 & 3.87 & 45.17 & 800 & 9 & & & & 40 \\
\hline I08572 & 0.05835 & 45.66 & 0.27 & 42.67 & 2817 & 2 & 1.62 & 11.8 & & 41,10 \\
\hline I10565 & 0.04311 & 44.81 & 0.11 & 41.07 & 535 & 5 & 1.98 & 11.17 & & 41,10 \\
\hline Mrk 231 & 0.04217 & 45.7 & -0.50 & 40.65 & 665 & 3 & 2.06 & 9.799 & & $41,2,3,4$ \\
\hline SDSSJ0149 & 0.567 & 46.94 & 2.60 & 44.25 & 1191 & 4.1 & 1.82 & 10.8 & & $42,43,44,45$ \\
\hline SDSSJ0210 & 0.54 & 46.16 & 2.62 & 43.62 & 560 & 7.5 & & 10.2 & & $42,43,44,45$ \\
\hline SDSSJ0319 & 0.626 & 46.44 & 2.32 & 43.76 & 934 & 7.5 & & 10.6 & & $42,43,44,45$ \\
\hline SDSSJ0321 & 0.643 & 46.51 & 2.30 & 43.75 & 946 & 11 & 1.28 & 11.2 & & $42,43,44,45$ \\
\hline SDSSJ0759 & 0.649 & 47.28 & 2.87 & 44.56 & 1250 & 7.5 & 1.64 & 11.3 & & $42,43,44,45$ \\
\hline SDSSJ0841 & 0.641 & 46.54 & 2.60 & 43.76 & 675 & 6.4 & & 10.9 & & $42,43,44,45$ \\
\hline SDSSJ0842 & 0.561 & 46.8 & 2.59 & 43.53 & 522 & 9 & 1.18 & 10.1 & & $42,43,44,45$ \\
\hline SDSSJ0858 & 0.454 & 47.23 & 2.79 & 44.24 & 939 & 5.6 & 2.08 & 10.6 & & $42,43,44,45$ \\
\hline SDSSJ1039 & 0.579 & 46.87 & 2.81 & 44.35 & 1046 & 5.8 & 1.57 & 10.6 & & $42,43,44,45$ \\
\hline SDSSJ1040 & 0.486 & 46.19 & 3.16 & 45.19 & 1821 & 7.6 & 1.59 & 10.7 & & $42,43,44,45$ \\
\hline SDSSJ1148 & 6.419 & 47.6 & 3.54 & 45.27 & 1300 & 8 & 3.00 & 11.58 & 10.3 & 46,47 \\
\hline \multicolumn{11}{|c|}{ BAL winds } \\
\hline SDSSJ1106 & 3.038 & 47.2 & 2.59 & 45.9 & 8000 & 0.3 & & & & 48,49 \\
\hline SDSSJ0838 & 2.043 & 47.5 & 2.48 & 45.79 & 8000 & 0.3 & & & & 50 \\
\hline SDSSJ0318 & 1.967 & 47.7 & 2.45 & 45.2 & 4200 & 11.5 & & & & 51 \\
\hline QSO2359 & 0.868 & 47.67 & 1.84 & 43.63 & 1380 & 3 & & & & 52,53 \\
\hline QSO1044 & 0.7 & 46.84 & 2.48 & 45.25 & 4300 & 1.7 & & & & 54 \\
\hline 3C 191 & 1.956 & 46.57 & 2.49 & 43.99 & 1000 & 28 & & & & 51 \\
\hline FIRST1214 & 0.6952 & 46.43 & 1.44 & 43.55 & 2000 & 0.03 & & & & 55 \\
\hline \multicolumn{11}{|c|}{ Ultra fast outflows } \\
\hline & & $\operatorname{erg~s}{ }^{-1}$ & $M_{\odot} / \mathrm{yr}$ & $\mathrm{erg} \mathrm{s}^{-1}$ & $\mathrm{~km} \mathrm{~s}^{-1}$ & $\mathrm{~cm}$ & $M_{\odot} / \mathrm{yr}$ & $M_{\odot}$ & $M_{\odot}$ & \\
\hline NGC 4151 & 0.003319 & 43.9 & -2.00 & 42.5 & $3.18 \times 10^{4}$ & $14.6-15.8$ & & & & 56 \\
\hline IC4329A & 0.016054 & 45.1 & -1.20 & 43.2 & $2.94 \times 10^{4}$ & $15.6-16.5$ & & & & 56 \\
\hline Mrk 509 & 0.034397 & 45.2 & -0.75 & 44.15 & $5.19 \times 10^{4}$ & $15.1-16.3$ & & & & 56 \\
\hline Mrk 509 & 0.034397 & 45.4 & -0.65 & 44.05 & $4.14 \times 10^{4}$ & $15.3-16.6$ & & & & 56 \\
\hline Ark 120 & 0.032713 & 45.5 & -0.70 & 44.65 & $8.61 \times 10^{4}$ & $14.8-17.9$ & & & & 56 \\
\hline
\end{tabular}

sources we find $\left\langle W 80 / v_{\max }\right\rangle=0.96 \pm 0.16$, i.e. $\mathrm{W} 80$ and $v_{\max }$ are very similar, on average within $4 \%$. We therefore conclude that by using the recipe W80/1.3 to estimate bulk wind velocities would produce mass outflow rates and kinetic energy rates smaller by $\sim 35 \%$ and by a factor $\sim 2.5$ respectively. Since we use the same recipe for all sources, of course using W80/1.3 instead of $v_{\max }$ will not change the results of the trend analysis.
$R_{\mathrm{OF}}$ is taken from the quoted literature. In most cases $R_{\mathrm{OF}}$ is taken as the maximum radius up to which high velocity gas is detected (baseline method). On the other hand, Carniani et al. (2015), evaluate a size of the ionised wind systematically lower than all other cases, because they adopt a different astrometric procedure. This gives rise to mass outflow rates higher than if they were calculated with the baseline method. For a few sources 
Table B.1. continued.

\begin{tabular}{|c|c|c|c|c|c|c|c|c|c|c|}
\hline Name $^{a}$ & Redshift & $\begin{array}{l}\log L_{\mathrm{bol}} \\
\mathrm{erg} \mathrm{s}^{-1}\end{array}$ & $\begin{array}{c}\log \dot{M}_{\mathrm{OF}} \\
M_{\odot} / \mathrm{yr}\end{array}$ & $\begin{array}{l}\log \dot{E}_{\mathrm{kin}} \\
\mathrm{erg} \mathrm{s}^{-1}\end{array}$ & $\begin{array}{c}v_{\max } \\
\mathrm{km} \mathrm{s}^{-1}\end{array}$ & $\underset{\operatorname{lpc}}{\log R_{\mathrm{OF}}}$ & $\begin{array}{c}\log S F R \\
M_{\odot} / \mathrm{yr}\end{array}$ & $\begin{array}{c}\log M_{*} \\
M_{\odot}\end{array}$ & $\begin{array}{c}\log M_{\text {gas }} \\
M_{\odot}\end{array}$ & REF \\
\hline Mrk 79 & 0.022189 & 44.9 & -0.45 & 43.95 & $2.76 \times 10^{4}$ & $15.3-16.5$ & & & & 56 \\
\hline NGC 4051 & 0.002336 & 43.3 & -2.65 & 40.95 & $1.11 \times 10^{4}$ & $14.7-15.9$ & & & & 56 \\
\hline Mrk 766 & 0.012929 & 44.2 & -1.80 & 42.5 & $2.46 \times 10^{4}$ & $13.8-17.2$ & & & & 56 \\
\hline Mrk 766 & 0.012929 & 44.4 & -1.70 & 42.6 & $2.64 \times 10^{4}$ & $13.7-16.1$ & & & & 56 \\
\hline Mrk 841 & 0.036422 & 44.9 & -0.90 & 43 & $1.65 \times 10^{4}$ & $15.8-18$ & & & & 56 \\
\hline 1H0419-577 & 0.104 & 45.6 & 0.50 & 44.7 & $2.37 \times 10^{4}$ & $16.3-17.9$ & & & & 56 \\
\hline Mrk 290 & 0.029577 & 44.6 & -0.55 & 44.35 & $4.89 \times 10^{4}$ & $14.8-16.7$ & & & & 56 \\
\hline Mrk 205 & 0.070846 & 45.2 & -0.20 & 44.2 & $3 \times 10^{4}$ & $16.1-16.2$ & & & & 56 \\
\hline PG1211+143 & 0.0809 & 45.3 & 0.50 & 45.3 & $4.53 \times 10^{4}$ & $15.3-18.5$ & & & & 56 \\
\hline MCG-5-23-16 & 0.008486 & 44.5 & -1.10 & 43.5 & $3.48 \times 10^{4}$ & $15-16.6$ & & & & 56 \\
\hline NGC 4507 & 0.011801 & 44.4 & -2.15 & 42.9 & $5.97 \times 10^{4}$ & $13.3-16.9$ & & & & 56 \\
\hline Mrk 231 & 0.04217 & 45.7 & 0.15 & 43.95 & $2.01 \times 10^{4}$ & $15.7-16.5$ & & & & 1 \\
\hline PDS456 & 0.184 & 47 & 1.30 & 46.3 & $1.05 \times 10^{5}$ & $16.2-16.2$ & & & & 57 \\
\hline I11119 & 0.189 & 46.2 & 0.20 & 45.3 & $7.65 \times 10^{4}$ & $14.2-15.9$ & & & & 58 \\
\hline APM08279 & 3.91 & 47.45 & 1.05 & 46.85 & $1.08 \times 10^{5}$ & $14-16$ & & & & 59 \\
\hline \multicolumn{11}{|c|}{ Warm absorbers } \\
\hline NGC 3783 & 0.009730 & 44.6 & -0.10 & 42.35 & 3000 & $17-19.1$ & & & & 56 \\
\hline NGC 3783 & 0.009730 & 44.4 & -0.55 & 41.55 & 2100 & $17.3-18.1$ & & & & 56 \\
\hline NGC 3783 & 0.009730 & 44.5 & -0.60 & 41.55 & 2100 & $17.3-18.1$ & & & & 56 \\
\hline NGC 3516 & 0.008836 & 44.8 & -1.00 & 41 & 1800 & $17.1-17.1$ & & & & 56 \\
\hline NGC 3516 & 0.008836 & 44.7 & -0.95 & 41.3 & 2400 & $16.8-16.6$ & & & & 56 \\
\hline NGC 3516 & 0.008836 & 44.6 & -1.00 & 41.5 & 3300 & $16.6-16.7$ & & & & 56 \\
\hline NGC 3516 & 0.008836 & 44.7 & -1.05 & 41.6 & 3900 & $16.4-16.7$ & & & & 56 \\
\hline Mrk 279 & 0.030451 & 45.1 & -0.60 & 41.5 & 2100 & $17.3-17.9$ & & & & 56 \\
\hline ESO323-G77 & 0.015014 & 45 & -0.35 & 42.25 & 3600 & $16.7-17$ & & & & 56 \\
\hline
\end{tabular}

integral field spectroscopy observation are not available and the size of the high velocity gas is estimated using off-centre spectra (Perna et al. 2015a; Bischetti et al. 2017). This method can detect only gas on relatively large scales, and therefore the relative mass outflow rates computed this way probably under-estimate the real rates.

For molecular winds, the mass of the outflowing gas is computed by converting $\mathrm{CO}$ luminosities into $\mathrm{H}_{2}$ masses. This is usually done by assuming a proper conversion factor $\alpha_{\mathrm{CO}}$, which can be a function of density, metallicity, gas distribution etc. (see Bolatto et al. 2013, for a review). We conservatively adopted $\alpha_{\mathrm{CO}}=0.8$ for our sample, mostly made by LIRGs and ULIRGs.

For ionised winds, the mass of the outflowing gas is calculated by using the following equations (Osterbrock \& Ferland 2006; Carniani et al. 2015):

$M_{[\mathrm{OIII}]}=4.0 \times 10^{7} M_{\odot}\left(\frac{C}{10^{\mathrm{O} / \mathrm{H}}}\right)\left(\frac{L_{[\mathrm{OIII}]}}{10^{44}}\right)\left(\frac{\left\langle n_{\mathrm{e}}\right\rangle}{10^{3}}\right)^{-1}$

and

$M_{\mathrm{H} \beta}=7.8 \times 10^{8} C\left(\frac{L_{\mathrm{H} \beta}}{10^{44}}\right)\left(\frac{\left\langle n_{\mathrm{e}}\right\rangle}{10^{3}}\right)^{-1}$

assuming that the contribution of star formation to the observed luminosities of the broad (wind) line emission is negligible, as in the literature quoted in Table B.1, and a gas temperature of $T=10^{4} \mathrm{~K}$. The $\mathrm{H}_{\beta}$ emissivity scales nearly linearly with the inverse of the temperature, so the mass of the outflow would be about twice for a temperature twice the one we consider here, for each given $\mathrm{H}_{\beta}$ luminosity. The [OIII] emissivity does not change much with the temperature for $T$ from a fraction to a few $10^{4} \mathrm{~K}$. Both $M_{\text {[OIII] }}$ and $M_{\mathrm{H} \beta}$ scale linearly with the average gas density $\left\langle n_{\mathrm{e}}\right\rangle$. This is often ill-defined, because it can be estimated only from the ratio of faint [SII] doublet. Genzel et al. (2014) use $\left\langle n_{\mathrm{e}}\right\rangle=80 \mathrm{~cm}^{-3}$ because this is the mean value in the disks and centres of star-forming galaxies. Nesvadba et al. (2006, 2008) found $\left\langle n_{\mathrm{e}}\right\rangle=240-570 \mathrm{~cm}^{-3}$ and $\left\langle n_{\mathrm{e}}\right\rangle=300-1000 \mathrm{~cm}^{-3}$ in two $z \sim 2$ radio galaxies. Harrison et al. (2014) find $\left\langle n_{\mathrm{e}}\right\rangle=$ $200-1000 \mathrm{~cm}^{-3}$ in a sample of low- $z$ AGN. Perna et al. (2015a) find $\left\langle n_{\mathrm{e}}\right\rangle=120 \mathrm{~cm}^{-3}$ in a $z=1.5 \mathrm{AGN}$. In the following analysis we adopt $\left\langle n_{\mathrm{e}}\right\rangle=200 \mathrm{~cm}^{-3}$ for all objects in the sample. For sources with $\mathrm{H}_{\beta}$ and $\mathrm{H}_{\alpha}$ measurements we used the gas mass evaluated from these lines. For sources with only [OIII] measurements we assume that the total ionised gas mass is 3 times the $M_{\text {[OIII] }}$ (this is the average of $M_{\mathrm{H} \beta} / M_{\text {[OIII] }}$ for the sources in our composite sample for which estimates of $M_{[\mathrm{OIII}]}$ and $M_{\mathrm{H} \beta}$ are simultaneously possible).

The largest uncertainty in the evaluation of molecular mass outflow rates is currently the size of the outflow. This uncertainty will likely greatly be reduced by future higher resolution ALMA and NOEMA observations. For ionised outflows, the largest uncertainties are the size of the outflow, the gas density and the $M_{\mathrm{H} \beta} / M_{\text {[OIII] }}$ ratio.

X-ray absorbers wind masses, outflow rates and kinetic power are even more uncertain than molecular and ionises gas masses outflow rates and kinetic power, due to the statistics of $\mathrm{X}$-ray spectra which is usually not excellent, and due to large systematic uncertainties in the evaluation on the size of the wind (only lower and upper limit can be derived from current, low resolution X-ray spectroscopy). The situation should greatly 
improve with the advent of high resolution micro-calorimeters in the X-ray bands (resolution of several thousands), which are planned for the Athena mission (Nandra et al. 2013).

\section{B.2. Host galaxies and AGN quantities}

We collected from literature for our AGN sample AGN bolometric luminosities, host galaxy star formation rates, stellar masses and total molecular gas masses (disk plus outflows), when available. We put particular care in searching and reporting star formation rates, stellar and gas masses relative to the size of the region interested by the wind $\left(R_{\mathrm{OF}}\right.$ in Table B.1). AGN bolometric luminosities are calculated by fitting to the observed UV to optical SEDs AGN + galaxy templates, and by converting the mid-infrared and or the X-ray luminosity by using a luminosity dependent bolometric corrections. When more than one estimate of AGN bolometric luminosity does exist (e.g. for bright local Seyfert galaxies), we used the one minimising the uncertainty due to a) obscuration of the active nucleus; b) contribution of the host galaxy to the observed luminosity. An additional source of scatter in AGN bolometric luminosity is due to AGN variability and the fact that most observations at different wavelengths are not simultaneous. We estimate that the total uncertainty on AGN bolometric luminosities can be the order of half a decade. This is still much smaller than the dynamic range in AGN bolometric luminosity investigated in this paper (five decades).

The SFR reported in Table B.1 are from far infrared photometry when possible. The AGN contribution to the far infrared band is negligible in most cases, but for the most luminous QSOs. Even in hyper-luminous QSO, Schneider et al. (2015) and Duras et al. (2017) found that $\lesssim$ half of the far infrared light is likely produced by dust in the galaxy disk illuminated by the AGN emission. The infrared computed SFR is not the instantaneous SFR but rather the conversion from the observed FIR luminosity produced by dust reprocessing of light from stars born hundreds of millions of years before. This SFR is therefore an upper limit to the on going SFR. Indeed, Davies et al. (2007) found that the on going SFR in the nuclei of Markarian 231 and NGC 1068 is probably very small, because of the small observed $\mathrm{Br} \gamma$ equivalent width within $0.1-0.5 \mathrm{kpc}$ from the active nucleus.

Stellar masses reported in Table B.1 are calculated by modelling optical-near-infrared galaxy SEDs with galaxy templates or by converting near infrared luminosities from IFU observations of nearby AGN host galaxies into stellar masses. 